\title{
On chiral effects in strain gradient elasticity
}

\author{
D. Ieşan ${ }^{a}$ and R. Quintanilla ${ }^{b}$ \\ ${ }^{a}$ Octav Mayer Institute of Mathematics, Romanian Academy, Bd. Carol I, nr. 8, \\ 700506 Iaşi, Romania, e-mail: iesan@uaic.ro \\ ${ }^{b}$ Department of Applied Mathematics, Polytechnic University of Catalonia, \\ Terrassa, Barcelona, Spain, e-mail: ramon.quintanilla@upc.edu
}

\begin{abstract}
This paper is concerned with the problem of uniformly loaded bars in strain gradient elasticity. We study the deformation of an isotropic chiral bar subjected to body forces, to tractions on the lateral surface and to resultant forces and moments on the ends. Examples of chiral materials include some auxetic materials, bones, some honeycomb structures, as well as composites with inclusions. The three-dimensional problem is reduced to the study of some generalized plane strain problems. The method is used to study the deformation of a uniformly loaded circular cylinder. New chiral effects are presented. The flexure of a chiral cylinder, in contrast with the case of achiral materials, is accompanied by extension and bending. The salient feature of the solution is that a uniform pressure acting on the lateral surface of a chiral circular elastic cylinder produces a twist around its axis.
\end{abstract}

Key words: Strain gradient elasticity, Chiral materials, Uniformly loaded bars, Flexure of the chiral bars, Circular cylinders

\section{Introduction}

The mechanical behaviour of chiral materials is of interest for the investigation of carbon nanotubes (see, e.g., Wang and Wang, 2007; Chandraseker et al. 2009; Askes and Aifantis, 2009; Zhang et al. 2010), auxetic materials (Spadoni and Ruzzene, 2012) and bones (Lakes et al. 1983; Park et al. 1986). The chiral effects cannot be described within classical elasticity (Lakes, 2001). The strain gradient theory of elasticity (Toupin, 1962; Mindlin, 1964; Mindlin and Eshel, 1968) is adequate to describe the deformation of chiral elastic solids (Papanicolopulos, 2011 and references therein).

In this paper we study the deformation of a homogeneous and isotropic chiral bar in the framework of the strain gradient elasticity. Mindlin (1964) pre-

Preprint submitted to Elsevier 9 October 2015 
sented three forms of the strain gradient elasticity. The relations among the three forms have been presented by Mindlin and Eshel (1968). Throughout this paper we will use the first form of the strain gradient elasticity. The three forms of the theory lead to the same displacement equations of motion for isotropic solids. The constitutive equations of isotropic chiral elastic solids in the strain gradient theory of elasticity have been established by Papanicolopulos (2011). In the present paper we consider the equilibrium of a right cylinder which is subjected to tractions on the lateral surface, to body forces, and to resultants forces and moments on the ends. We assume that the body forces and the lateral tractions are independent of the axial coordinate. The three-dimensional problem is reduced to the study of some two-dimensional problems. The paper is structured as follows. First, we present the basic equations of isotropic chiral elastic solids and formulate the problem of uniformly loaded cylinders. Then, we define the generalized plane strain problem and introduce four auxiliary plane problems necessary to investigate the deformation of loaded cylinders. In the following section we establish the solution of the problem of uniformly loaded bar. In the classical elasticity this problem is known as Almansi-Michell problem and was studied in various works (Khatiashvili, 1983; Ieşan and Quintanilla, 2007; Ieşan, 2009). We show that the body forces and the tractions on the lateral surface produce extension, torsion, flexure, bending by terminal couples and a plane strain. The solution is used to study the deformation of a circular cylinder. We present new chiral effects. It is shown that the flexure of a chiral cylinder, in contrast with the case of achiral bars, is accompanied by extension and bending. The salient feature of the solution is that a uniform pressure acting on the lateral surface of a chiral circular cylinder produces a twist around its axis.

\section{Basic equations}

In this section we present the basic equation of isotropic chiral elastic solids in the first strain-gradient theory and the formulation of the problem of uniformly loaded cylinders. We consider a body that in undeformed state occupies the region $B$ of euclidean three-dimensional space and is bounded by the surface $\partial B$. We refer the deformation of the body to a fixed system of rectangular axes $O x_{k},(k=1,2,3)$. Let $\mathbf{n}$ be the outward unit normal of $\partial B$. Letters in boldface stand for tensors of an order $p \geq 1$, and if $\boldsymbol{v}$ has the order $p$, we write $v_{i j \ldots k}$ ( $p$ subscripts) for the components of $\boldsymbol{v}$ in the Cartesian coordinate system. We shall employ the usual summation and differentiation conventions: Latin subscripts (unless otherwise specified) are understood to range over the integers $(1,2,3)$, whereas Greek subscripts to the range $(1,2)$, summation over repeated subscripts is implied and subscripts preceded by a comma denote partial differentiation with respect to the corresponding Cartesian coordinate. 
We assume that $B$ is a bounded region with Lipschitz boundary $\partial B$, consisting of a finite number of smooth surfaces. Let $\Gamma_{p}$ be the intersection of two adjoined smooth surfaces and $C=\cup \Gamma_{p}$. We assume that $B$ is occupied by a homogeneous and isotropic chiral elastic solid. Let $\boldsymbol{u}$ be the displacement vector field. The strain measures are given by (Mindlin and Eshel, 1968)

$$
e_{i j}=\frac{1}{2}\left(u_{i, j}+u_{j, i}\right), \quad \kappa_{i j k}=u_{k, i j}
$$

The constitutive equations for isotropic chiral elastic solids are (Mindlin and Eshel, 1968; Papanicolopulos, 2011).

$$
\begin{aligned}
\tau_{i j} & =\lambda e_{r r} \delta_{i j}+2 \mu e_{i j}+f\left(\varepsilon_{i k m} \kappa_{j k m}+\varepsilon_{j k m} \kappa_{i k m}\right), \\
\mu_{i j k} & =\frac{1}{2} \alpha_{1}\left(\kappa_{r r i} \delta_{j k}+2 \kappa_{k r r} \delta_{i j}+\kappa_{r r j} \delta_{i k}\right)+ \\
& +\alpha_{2}\left(\kappa_{i r r} \delta_{j k}+\kappa_{j r r} \delta_{i k}\right)+2 \alpha_{3} \kappa_{r r k} \delta_{i j}+ \\
& +2 \alpha_{4} \kappa_{i j k}+\alpha_{5}\left(\kappa_{k j i}+\kappa_{k i j}\right)+f\left(\varepsilon_{i k s} e_{j s}+\varepsilon_{j k s} e_{i s}\right),
\end{aligned}
$$

where $\tau_{i j}$ is the stress tensor, $\mu_{i j k}$ is the double stress tensor, $\delta_{i j}$ is the Kronecker delta, $\varepsilon_{i j k}$ is the alternating symbol and $\lambda, \mu, \alpha_{s},(s=1,2, \ldots, 5)$, and $f$ are constitutive constants. The terms from (2) which contain the coefficient $f$ represent the chiral part of the constitutive equations. The constitutive equations for chiral solids have been established by Papanicolopulos (2011). In the case of a centrosymmetric (achiral) material the coefficient $f$ is equal to zero. The equilibrium equations are

$$
\tau_{j i, j}-\mu_{s j i, s j}+F_{i}=0
$$

where $F_{i}$ is the body force per unit volume. Following Toupin (1962) and Mindlin (1964) we introduce the functions $P_{i}, R_{i}$ and $Q_{i}$ by

$$
\begin{aligned}
& P_{i}=\left(\tau_{k i}-\mu_{r k i, r}\right) n_{k}-D_{j}\left(n_{r} \mu_{r j i}\right)+\left(D_{k} n_{k}\right) n_{s} n_{p} \mu_{s p i} \\
& R_{i}=\mu_{r s i} n_{r} n_{s}, \quad Q_{i}=\left\langle\mu_{p j i} n_{p} n_{q}\right\rangle \varepsilon_{j r q} s_{r}
\end{aligned}
$$

where $D_{i}$ are the components of the surface gradient, $D_{i}=\left(\delta_{i k}-n_{i} n_{k}\right) \partial / \partial x_{k}$, $s_{k}$ are the components of the unit vector tangent to $C$, and $\langle g\rangle$ denotes the difference of limits of $g$ from both sides of $C$. We denote by $\bar{B}$ the closure of $B$. We say that the vector field $u_{j}$ is an admissible displacement field on $B$ provided $u_{j} \in C^{4}(B)$. An admissible system of stresses on $\bar{B}$ is an ordered array of function $\left(\tau_{i j}, \mu_{p q r}\right)$ with the following properties: (i) $\tau_{i j} \in C^{1}(\bar{B}), \mu_{i j k} \in$ $C^{2}(\bar{B})$; (ii) $\tau_{i j}=\tau_{j i}, \mu_{i j k}=\mu_{j k i}$. By an admissible state on $\bar{B}$ we mean an ordered array of fields $A=\left(u_{i}, e_{i j}, \kappa_{i j k}, \tau_{i j}, \mu_{i j k}\right)$ with the properties: (i) $u_{i}$ is an admissible displacement field on $\bar{B}$; (ii) $e_{i j} \in C^{1}(\bar{B}), \kappa_{i j k} \in C^{2}(\bar{B}), e_{i j}=e_{j i}$, $\kappa_{i j k}=\kappa_{j i k}$; (iii) $\left(\tau_{i j}, \mu_{i j k}\right)$ is an admissible system of stresses on $\bar{B}$. By an external data system on $\bar{B}$ we mean an ordered array $L=\left(F_{i}, \widetilde{P}_{i}, \widetilde{R}_{i}, \widetilde{Q}_{i}\right)$ with the properties: (i) $F_{i}$ is continuous on $\bar{B}$; (ii) $\widetilde{P}_{i}$ and $\widetilde{R}_{i}$ are piecewise regular on 
$\partial B$; (iii) $\widetilde{Q}_{i}$ is piecewise regular on $C$. We say that $A=\left(u_{i}, e_{i j}, \kappa_{i j k}, \tau_{i j}, \mu_{i j k}\right)$ is an elastic state corresponding to the body force $F_{k}$ if $A$ is an admissible state that satisfies the equations (1)-(3) on $B$. The traction problem of elastostatics consists in finding an elastic state that corresponds to the body force $F_{i}$ and satisfies the boundary conditions

$$
P_{i}=\widetilde{P}_{i}, \quad R_{i}=\widetilde{R}_{i} \text { on } \partial B \backslash C, Q_{i}=\widetilde{Q}_{i} \text { on } C,
$$

where $\widetilde{P}_{i}, \widetilde{R}_{i}$ and $\widetilde{Q}_{i}$ are prescribed functions.

The potential energy density for isotropic chiral materials is given by

$$
\begin{aligned}
W & =\frac{1}{2} \lambda e_{r r} e_{j j}+\mu e_{i j} e_{i j}+\alpha_{1} \kappa_{i i k} \kappa_{k j j}+ \\
& +\alpha_{2} \kappa_{i j j} \kappa_{i r r}+\alpha_{3} \kappa_{i i r} \kappa_{j j r}+\alpha_{4} \kappa_{i j k} \kappa_{i j k}+\alpha_{5} \kappa_{i j k} \kappa_{k j i}+2 f \varepsilon_{i k m} e_{i j} \kappa_{k j m} .
\end{aligned}
$$

In what follows we assume that the elastic potential is a positive definite quadratic form in the variables $e_{i j}$ and $\kappa_{i j k}$. The restrictions imposed by this assumption on the constitutive coefficients have been presented by Mindlin and Eshel (1968) and Papanicolopulos (2011). The necessary and sufficient conditions for the existence of a solution of the traction problem are (Hlavacek and Hlavacek, 1969)

$$
\begin{aligned}
& \int_{B} F_{i} d v+\int_{\partial B} \widetilde{P}_{i} d a+\int_{C} \widetilde{Q}_{i} d s=0, \\
& \int_{B} \varepsilon_{i j k} x_{j} F_{k} d v+\int_{\partial B} \varepsilon_{i j k}\left(x_{j} \widetilde{P}_{k}+n_{j} \widetilde{R}_{k}\right) d a+\int_{C} \varepsilon_{i j k} x_{j} \widetilde{Q}_{k} d s=0 .
\end{aligned}
$$

We assume that the region $B$ from here on refers to the interior of a right cylinder of length $h$ with the cross-section $\Sigma$ and the lateral boundary $\Pi$. Let $\Gamma$ be the boundary of $\Sigma$. The Cartesian coordinate frame is supposed to be chosen in such a way that $x_{3}$-axis is parallel to the generators of $B$ and the $x_{1} O x_{2}$ plane contains one of terminal cross-sections. We denote by $\Sigma_{1}$ and $\Sigma_{2}$, respectively, the cross-section located at $x_{3}=0$ and $x_{3}=h$. We denote by $\Gamma_{\alpha}$ the boundary of the cross-section $\Sigma_{\alpha}$. We assume that the lateral surface $\Pi$ is smooth, so that $Q_{i}$ is equal to zero on $\Pi$. We shall use Saint-Venant's approach of the problem of elastic cylinders which is based on a relaxed statement in which the pointwise assignment of the terminal tractions is replaced by prescribing the corresponding resultant force and resultant moment. We assume that the cylinder is subjected to body forces, to tractions on the lateral surface and to appropriate global conditions on the ends. The conditions on the lateral boundary are

$$
P_{i}=\widetilde{P}_{i}, \quad R_{i}=\widetilde{R}_{i} \text { on } \Pi
$$


Let $\mathcal{F}=\left(\mathcal{F}_{1}, \mathcal{F}_{2}, \mathcal{F}_{3}\right)$ and $\mathcal{M}=\left(M_{1}, M_{2}, M_{3}\right)$ be prescribed vectors representing the resultant force and the resultant moment about $O$ of the tractions acting on $\Sigma_{1}$. On $\Sigma_{2}$ there are tractions applied so as to satisfy the equilibrium conditions of the body. For the end located at $x_{3}=0$ we have the following conditions

$$
\begin{aligned}
& \int_{\Sigma_{1}} P_{\alpha} d a+\int_{\Gamma_{1}} Q_{\alpha} d s=\mathcal{F}_{\alpha}, \\
& \int_{\Sigma_{1}} P_{3} d a+\int_{\Gamma_{1}} Q_{3} d s=\mathcal{F}_{3}, \\
& \int_{\Sigma_{1}}\left(x_{\alpha} P_{3}+R_{\alpha}\right) d a+\int_{\Gamma_{1}} x_{\alpha} Q_{3} d s=\varepsilon_{\beta \alpha 3} M_{\beta}, \\
& \int_{\Sigma_{1}} \varepsilon_{\alpha \beta 3} x_{\alpha} P_{\beta} d a+\int_{\Gamma_{1}} \varepsilon_{\alpha \beta 3} x_{\alpha} Q_{\beta} d s=M_{3} .
\end{aligned}
$$

In the theory of uniformly loaded bars the loads $F_{i}, \widetilde{P}_{i}$ and $\widetilde{R}_{i}$ are prescribed functions which are independent of the axial coordinate,

$$
F_{j, 3}=0, \quad \widetilde{P}_{j, 3}=0, \quad \widetilde{R}_{j, 3}=0 .
$$

The problem of uniformly loaded bars consists in finding the functions $u_{i}$ which satisfy Eqs. (1)-(3) on $B$, the conditions (8) on the lateral surface, and the conditions (9)-(12) on the end $\Sigma_{1}$, when the functions $F_{i}, \widetilde{P}_{i}, \widetilde{R}_{i}$ and the constants $\mathcal{F}_{j}, M_{j}, \lambda, \mu, \alpha_{s},(s=1,2, \ldots, 5)$, and $f$ are given.

\section{Preliminaries}

In this section we define the generalized plane strain of the cylinder $B$ and introduce four auxiliary plane problems necessary to investigate the problem of uniformly loaded bars. Let $A=\left(u_{i}, e_{i j}, \kappa_{i j k}, \tau_{i j}, \mu_{i j k}\right)$ be an elastic state on the cylinder $B$. Then $A$ is a state of generalized plane strain provided

$$
u_{i}=u_{i}\left(x_{1}, x_{2}\right), \quad\left(x_{1}, x_{2}\right) \in \Sigma .
$$

The relations (1), (2) and (19) imply that $e_{i j}, \kappa_{i j k}, \tau_{i j}$ and $\mu_{i j k}$ are all independent of the axial coordinate. The strain measures (1) reduce to

$$
2 e_{\alpha \beta}=u_{\alpha, \beta}+u_{\beta, \alpha}, 2 e_{\alpha 3}=u_{3, \alpha}, \kappa_{\alpha \beta j}=u_{j, \alpha \beta}
$$

and $e_{33}=0, \kappa_{k 3 i}=0$. The constitutive equations become

$$
\begin{aligned}
\tau_{\alpha \beta} & =\lambda e_{\rho \rho} \delta_{a \beta}+2 \mu e_{\alpha \beta}+f\left(\varepsilon_{\alpha \rho 3} \kappa_{\beta \rho 3}+\varepsilon_{\beta \rho 3} \kappa_{\alpha \rho 3}\right), \\
\tau_{\alpha 3} & =2 \mu e_{\alpha 3}+f \varepsilon_{\rho \beta 3} \kappa_{\alpha \rho \beta}, \\
\mu_{\alpha \beta \gamma} & =\frac{1}{2} \alpha_{1}\left(\kappa_{\rho \rho \alpha} \delta_{\beta \gamma}+2 \kappa_{\gamma \rho \rho} \delta_{\alpha \beta}+\kappa_{\rho \rho \beta} \delta_{\alpha \gamma}\right)+
\end{aligned}
$$




$$
\begin{aligned}
& +\alpha_{2}\left(\kappa_{\alpha \rho \rho} \delta_{\beta \gamma}+\kappa_{\beta \rho \rho} \delta_{\alpha \gamma}\right)+2 \alpha_{3} \kappa_{\rho \rho \gamma} \delta_{\alpha \beta}+ \\
& +2 \alpha_{4} \kappa_{\alpha \beta \gamma}+\alpha_{5}\left(\kappa_{\gamma \beta \alpha}+\kappa_{\gamma \alpha \beta}\right)+f\left(\varepsilon_{\alpha \gamma 3} e_{\beta 3}+\varepsilon_{\beta \gamma 3} e_{\alpha 3}\right), \\
\mu_{\alpha \beta 3} & =2 \alpha_{3} \kappa_{\rho \rho 3} \delta_{\alpha \beta}+2 \alpha_{4} \kappa_{\alpha \beta 3}+f\left(\varepsilon_{\rho \alpha 3} e_{\beta \rho}+\varepsilon_{\rho \beta 3} e_{\alpha \rho}\right),
\end{aligned}
$$

and

$$
\begin{aligned}
& \tau_{33}=\lambda e_{\rho \rho}, \mu_{3 \alpha \beta}=\frac{1}{2} \alpha_{1} \kappa_{\rho \rho 3} \delta_{\alpha \beta}+\alpha_{5} \kappa_{\beta \alpha 3}+f \varepsilon_{\beta \rho 3} e_{\alpha \rho}, \\
& \mu_{3 \alpha 3}=\frac{1}{2} \alpha_{1} \kappa_{\rho \rho \alpha}+\alpha_{2} \kappa_{\alpha \rho \rho}+f \varepsilon_{\rho \alpha 3} e_{3 \rho}, \\
& \mu_{33 \alpha}=\alpha_{1} \kappa_{\alpha \rho \rho}+2 \alpha_{3} \kappa_{\rho \rho \alpha}+2 f \varepsilon_{\alpha \rho 3} e_{3 \rho}, \quad \mu_{333}=\left(\alpha_{1}+2 \alpha_{3}\right) \kappa_{\rho \rho 3} .
\end{aligned}
$$

The equations of equilibrium reduce to

$$
\tau_{\beta j, \beta}-\mu_{\rho \nu j, \rho \nu}+F_{j}=0 \text { on } \Sigma .
$$

It follows from (4) that in the case of a generalized plane strain we have the following relations on the lateral surface $\Pi$

$$
\begin{aligned}
& P_{i}=\left(\tau_{\beta i}-\mu_{\rho \beta i, \rho}\right) n_{\beta}-D_{\rho}\left(n_{\beta} \mu_{\beta \rho i}\right)+\left(D_{\rho} n_{\rho}\right) n_{\beta} n_{\nu} \mu_{\beta \nu i}, \\
& R_{i}=\mu_{\rho \nu i} n_{\rho} n_{\nu} .
\end{aligned}
$$

The conditions on the lateral surface reduce to

$$
P_{i}=\widetilde{P}_{i}, \quad R_{i}=\widetilde{R}_{i} \text { on } \Gamma_{1} .
$$

The generalized plane strain problem consists in finding an elastic state on $\bar{B}$ which satisfies the geometrical equations (15), the constitutive equations (16) and the equilibrium equations (17) on $\Sigma$, and the boundary conditions (19) on $\Gamma$. We assume that $F_{i}, \widetilde{P}_{i}$ and $\widetilde{R}_{i}$ are functions of class $C^{\infty}$, and that $\Sigma$ is $C^{\infty}$-smooth. The functions $\tau_{33}, \mu_{3 \beta i}$ and $\mu_{33 i}$ can be determined after the displacement field is found. In view of (15) and (16), the equations of equilibrium (17) can be expressed in terms of the functions $u_{k}$ in the form

$$
\begin{aligned}
& \mu \Delta u_{\alpha}+(\lambda+\mu) u_{\beta, \beta \alpha}-2\left(\alpha_{3}+\alpha_{4}\right) \Delta \Delta u_{\alpha}- \\
& -2\left(\alpha_{1}+\alpha_{2}+\alpha_{5}\right) \Delta u_{\beta, \beta \alpha}+2 f \varepsilon_{\alpha \beta 3} \Delta u_{3, \beta}+F_{\alpha}=0, \\
& \mu \Delta u_{3}-2\left(\alpha_{3}+\alpha_{4}\right) \Delta \Delta u_{3}+2 f \varepsilon_{\rho \nu 3} \Delta u_{\nu, \rho}+F_{3}=0, \text { on } \Sigma,
\end{aligned}
$$

where $\Delta$ is the Laplacian operator. By using the results presented by Hlavacek and Hlavacek (1969) we can show that the generalized plane strain problem has a solution if and only if

$$
\begin{aligned}
& \int_{\Sigma} F_{k} d a+\int_{\Gamma} \widetilde{P}_{k} d s=0, \\
& \int_{\Sigma} \varepsilon_{3 \alpha \beta} x_{\alpha} F_{\beta} d a+\int_{\Gamma_{1}} \varepsilon_{3 \alpha \beta}\left(x_{\alpha} \widetilde{P}_{\beta}+n_{\alpha} \widetilde{R}_{\beta}\right) d s=0 .
\end{aligned}
$$


In what follows we will use four special problems of generalized plane strain, denoted by $A^{(k)},(k=1,2,3,4)$. In the problem $A^{(1)}$ the external data system is $\left(F_{i}^{(1)}, \widetilde{P}_{i}^{(1)}, \widetilde{R}_{i}^{(1)}\right)$ where

$$
\begin{aligned}
& F_{i}^{(1)}=\lambda \delta_{1 i}, \widetilde{P}_{1}^{(1)}=-\lambda x_{1} n_{1}+\left(\alpha_{1}-2 \alpha_{2}\right) \varepsilon_{3 \alpha \nu}\left(n_{1} n_{2}\right)_{, \nu} n_{\alpha}, \\
& \widetilde{P}_{2}^{(1)}=-\lambda x_{1} n_{2}+\frac{1}{2}\left(\alpha_{1}-2 \alpha_{2}\right) \varepsilon_{3 \alpha \nu}\left(n_{1}^{2}-n_{2}^{2}\right)_{, \alpha} n_{\nu}, \\
& \widetilde{P}_{3}^{(1)}=2 f n_{2}, \widetilde{R}_{1}^{(1)}=2 \alpha_{3}-\alpha_{1}+\left(\alpha_{1}-2 \alpha_{2}\right) n_{1}^{2}, \\
& \widetilde{R}_{2}^{(1)}=\left(\alpha_{1}-2 \alpha_{2}\right) n_{1} n_{2}, \widetilde{R}_{3}^{(1)}=0 .
\end{aligned}
$$

The problem $A^{(2)}$ is characterized by the following loading

$$
\begin{aligned}
& F_{i}^{(2)}=\lambda \delta_{2 i}, \widetilde{P}_{1}^{(2)}=-\lambda x_{2} n_{1}+\frac{1}{2}\left(\alpha_{1}-2 \alpha_{2}\right) \varepsilon_{3 \alpha \nu}\left(n_{1}^{2}-n_{2}^{2}\right)_{, \alpha} n_{\nu}, \\
& \widetilde{P}_{2}^{(2)}=-\lambda x_{2} n_{2}+\left(\alpha_{1}-2 \alpha_{2}\right) \varepsilon_{3 \alpha \nu}\left(n_{1} n_{2}\right)_{, \nu} n_{\alpha}, \widetilde{P}_{3}^{(2)}=-2 f n_{1}, \\
& \widetilde{R}_{1}^{(2)}=\left(\alpha_{1}-2 \alpha_{2}\right) n_{1} n_{2}, \widetilde{R}_{2}^{(2)}=2 \alpha_{3}-\alpha_{1}+\left(\alpha_{1}-2 \alpha_{2}\right) n_{2}^{2}, \widetilde{R}_{3}^{(2)}=0 .
\end{aligned}
$$

In the problem $A^{(3)}$ the body force and the boundary data are given by

$$
F_{i}^{(3)}=0, \widetilde{P}_{\alpha}^{(3)}=-\lambda n_{\alpha}, \widetilde{P}_{3}^{(3)}=0, \widetilde{R}_{i}^{(3)}=0
$$

The problem $A^{(4)}$ corresponds to the following external data

$$
\begin{aligned}
& F_{i}^{(4)}=0, \widetilde{P}_{1}^{(4)}=\frac{1}{2} f\left[5 n_{1}+D_{1}\left(x_{2} n_{2}\right)+D_{2}\left(x_{2} n_{1}-2 x_{1} n_{2}\right)-\right. \\
& \left.-2\left(x_{2} n_{1} n_{2}-x_{1} n_{2}^{2}\right)\left(D_{\rho} n_{\rho}\right)\right], \\
& \widetilde{P}_{2}^{(4)}=\frac{1}{2} f\left[5 n_{2}+D_{1}\left(x_{1} n_{2}-2 x_{2} n_{1}\right)+D_{2}\left(x_{1} n_{1}\right)-\right. \\
& \left.-2\left(x_{1} n_{1} n_{2}-x_{2} n_{1}^{2}\right)\left(D_{\rho} n_{\rho}\right)\right], \quad \widetilde{P}_{3}^{(4)}=\mu \varepsilon_{3 \beta \rho} x_{\rho} n_{\beta}, \\
& \widetilde{R}_{1}^{(4)}=f\left(x_{1} n_{2}^{2}-x_{2} n_{1} n_{2}\right), \quad \widetilde{R}_{2}^{(4)}=f\left(x_{2} n_{1}^{2}-x_{1} n_{1} n_{2}\right), \quad \widetilde{R}_{3}^{(4)}=0 .
\end{aligned}
$$

The necessary and sufficient conditions (21) for the existence of the solution are satisfied for each boundary value problem $A^{(k)},(k=1,2,3,4)$. We denote by $u_{i}^{(k)}, e_{i j}^{(k)}, \kappa_{p q r}^{(k)}, \tau_{i j}^{(k)}$ and $\mu_{p q r}^{(k)}$ the displacement, the strain measures, the stress tensor and the double stress tensor in the problem $A^{(k)},(k=1,2,3,4)$, respectively. Let us introduce the notations

$$
\begin{aligned}
P_{i}^{(k)} & =\left(\tau_{\beta i}^{(k)}-\mu_{\rho \beta i, \rho}^{(k)}\right) n_{\beta}-D_{\rho}\left(n_{\beta} \mu_{\beta \rho i}^{(k)}\right)+\left(D_{\rho} n_{\rho}\right) \mu_{\beta \nu i}^{(k)} n_{\beta} n_{\nu}, \\
R_{i}^{(k)} & =\mu_{\rho \nu i}^{(k)} n_{\rho} n_{\nu} .
\end{aligned}
$$

Clearly, the functions $u_{i}^{(k)}, e_{i j}^{(k)}, \kappa_{p q r}^{(k)}, \tau_{i j}^{(k)}$ and $\mu_{p q r}^{(k)}$ satisfy the geometrical equations

$$
2 e_{\alpha \beta}^{(k)}=u_{\alpha, \beta}^{(k)}+u_{\beta, \alpha}^{(k)}, 2 e_{\alpha 3}^{(k)}=u_{3, \alpha}^{(k)}, \kappa_{\alpha \beta j}^{(k)}=u_{j, \alpha \beta}^{(k)},
$$


the constitutive equations

$$
\begin{aligned}
& \tau_{\alpha \beta}^{(k)}=\lambda e_{\rho \rho}^{(k)} \delta_{\alpha \beta}+2 \mu e_{\alpha \beta}^{(k)}+f\left(\varepsilon_{\alpha \rho 3} \kappa_{\beta \rho 3}^{(k)}+\varepsilon_{\beta \rho 3} \kappa_{\alpha \rho 3}^{(k)}\right), \\
& \tau_{\alpha 3}^{(k)}=2 \mu e_{\alpha 3}^{(k)}+f \varepsilon_{\rho \beta 3} \kappa_{\alpha \rho \beta}^{(k)}, \\
& \mu_{\alpha \beta \gamma}^{(k)}=\frac{1}{2} \alpha_{1}\left(\kappa_{\rho \rho \alpha}^{(k)} \delta_{\beta \gamma}+2 \kappa_{\gamma \rho \rho}^{(k)} \delta_{\alpha \beta}+\kappa_{\rho \rho \beta}^{(k)} \delta_{\alpha \gamma}\right)+ \\
& +\alpha_{2}\left(\kappa_{\alpha \rho \rho}^{(k)} \delta_{\beta \gamma}+\kappa_{\beta \rho \rho}^{(k)} \delta_{\alpha \gamma}\right)+2 \alpha_{3} \kappa_{\rho \rho \gamma}^{(k)} \delta_{\alpha \beta}+ \\
& +2 \alpha_{4} \kappa_{\alpha \beta \gamma}^{(k)}+\alpha_{5}\left(\kappa_{\gamma \beta \alpha}^{(k)}+\kappa_{\gamma \alpha \beta}^{(k)}\right)+f\left(\varepsilon_{\alpha \gamma 3} e_{\beta 3}^{(k)}+\varepsilon_{\beta \gamma 3} e_{\alpha 3}^{(k)}\right), \\
& \mu_{\alpha \beta 3}^{(k)}=2 \alpha_{3} \kappa_{\rho \rho 3}^{(k)} \delta_{\alpha \beta}+2 \alpha_{4} \kappa_{\alpha \beta 3}^{(k)}+f\left(\varepsilon_{\rho \alpha 3} e_{\beta \rho}^{(k)}+\varepsilon_{\rho \beta 3} e_{\alpha \rho}^{(k)}\right),
\end{aligned}
$$

and the equilibrium equations

$$
\tau_{\beta j, \beta}^{(k)}-\mu_{\rho \nu j, \rho \nu}^{(k)}+F_{j}^{(k)}=0
$$

on $\Sigma$, and the boundary conditions

$$
P_{i}^{(k)}=\widetilde{P}_{i}^{(k)}, \quad R_{i}^{(k)}=\widetilde{R}_{i}^{(k)} \text { on } \Gamma_{1}
$$

where $F_{i}^{(k)}, \widetilde{P}_{i}^{(k)}$ and $\widetilde{R}_{i}^{(k)},(k=1,2,3,4)$ are defined by $(22)-(25)$.

We have introduced the auxiliary plane problems $A^{(k)},(k=1,2,3,4)$, in this section in order to simplify the presentation of the method. In fact, we have obtained these problems when we have imposed that the equations of equilibrium (3) and the boundary conditions (8) be satisfied by the stress tensor and the double stress tensor given by (37) and (39). If we impose that the coefficients of the constants $a_{k}$ which appear in the equilibrium equations and boundary conditions be equal to zero, then we obtain the body forces and the boundary data that characterize the problems $A^{(k)}$.

\section{Solution of the problem}

In this section we present the solution to the problem of uniformly loaded cylinders. It is known (Ieşan, 1986a; Ieşan, 2009) that the solution of the problem of extension, bending and torsion can be found in the class of displacement vector fields $\boldsymbol{u}$ with the property that $\boldsymbol{u}_{3}$ is a rigid displacement. The solution $\boldsymbol{u}$ of the flexure problem has the property that $\boldsymbol{u}_{, 3}$ is a solution of a problem of extension, bending and torsion. The solution of the problem of uniformly loaded cylinders can be found in the class of displacement vector fields $\boldsymbol{u}$ with the property that $\boldsymbol{u}_{33}$ is a solution of Saint-Venant's problem (Ieşan, 1986b). This result has been established in the classical theory but it also holds in the theory of elastic solids with microstructure (Ieşan, 2009). We 
seek the solution of the problem formulated in Section 2 in the form

$$
\begin{aligned}
u_{\alpha} & =-\frac{1}{2} a_{\alpha} x_{3}^{2}-\frac{1}{6} b_{\alpha} x_{3}^{3}-\frac{1}{24} c_{\alpha} x_{3}^{4}+\varepsilon_{3 \beta \alpha}\left(a_{4} x_{3}+\frac{1}{2} b_{4} x_{3}^{2}+\frac{1}{6} c_{4} x_{3}^{3}\right) x_{\beta} \\
& +\sum_{k=1}^{4}\left(a_{k}+b_{k} x_{3}+\frac{1}{2} c_{k} x_{3}^{2}\right) u_{\alpha}^{(k)}+w_{\alpha}\left(x_{1}, x_{2}\right)+x_{3} v_{\alpha}\left(x_{1}, x_{2}\right), \\
u_{3} & =\left(a_{1} x_{1}+a_{2} x_{2}+a_{3}\right) x_{3}+\frac{1}{2}\left(b_{1} x_{1}+b_{2} x_{2}+b_{3}\right) x_{3}^{2}+\frac{1}{6}\left(c_{1} x_{1}+c_{2} x_{2}+c_{3}\right) x_{3}^{3} \\
& +\sum_{s=1}^{4}\left(a_{s}+b_{s} x_{3}+\frac{1}{2} c_{s} x_{3}^{2}\right) u_{3}^{(s)}+w_{3}\left(x_{1}, x_{2}\right)+x_{3} v_{3}\left(x_{1}, x_{2}\right),
\end{aligned}
$$

where $u_{i}^{(k)}$ are the displacement in the problem $A^{(k)}, w_{j}$ and $v_{j}$ are unknown functions, and $a_{k}, b_{k}$ and $c_{k},(k=1,2,3,4)$, are unknown constants. Let us note that the displacement vector $\boldsymbol{u}$ given by (31) has the property that its partial derivative of third order with respect to axial coordinate is a rigid displacement.

We denote by $\gamma_{i j}$ and $\eta_{\alpha \beta k}$ the strain measures in the generalized plane strain corresponding to the displacement $w_{j}$,

$$
\gamma_{\alpha \beta}=\frac{1}{2}\left(w_{\alpha, \beta}+w_{\beta, \alpha}\right), 2 \gamma_{\alpha 3}=w_{3, \alpha}, \quad \eta_{\alpha \beta k}=w_{k, \alpha \beta}
$$

Let us consider a generalized plane strain in which the components of displacement vector are the functions $v_{k}$. The strain measures in this problem are defined by

$$
\xi_{\alpha \beta}=\frac{1}{2}\left(v_{\alpha, \beta}+v_{\beta, \alpha}\right), 2 \xi_{\alpha 3}=v_{3, \alpha}, \zeta_{\alpha \beta k}=v_{k, \alpha \beta} .
$$

It follows from (1) and (31)-(33) that

$$
\begin{aligned}
e_{\alpha \beta} & =\sum_{k=1}^{4}\left(a_{k}+b_{k} x_{3}+\frac{1}{2} c_{k} x_{3}^{2}\right) e_{\alpha \beta}^{(k)}+\gamma_{\alpha \beta}+x_{3} \xi_{\alpha \beta} \\
e_{\alpha 3} & =\frac{1}{2} \varepsilon_{3 \beta \alpha}\left(a_{4}+b_{4} x_{3}+\frac{1}{2} c_{4} x_{3}^{2}\right) x_{\beta}+\sum_{k=1}^{4}\left(a_{k}+b_{k} x_{3}+\frac{1}{2} c_{k} x_{3}^{2}\right) e_{3 \alpha}^{(k)} \\
& +\gamma_{\alpha 3}+x_{3} \xi_{\alpha 3}+\frac{1}{2} \sum_{k=1}^{4}\left(b_{k}+c_{k} x_{3}\right) u_{\alpha}^{(k)}+\frac{1}{2} v_{\alpha} \\
e_{33} & =a_{1} x_{1}+a_{2} x_{2}+a_{3}+\left(b_{1} x_{1}+b_{2} x_{2}+b_{3}\right) x_{3}+\frac{1}{2}\left(c_{1} x_{1}+\right. \\
& \left.+c_{2} x_{2}+c_{3}\right) x_{3}^{2}+\sum_{k=1}^{4}\left(b_{k}+c_{k} x_{3}\right) u_{3}^{(k)}+v_{3} \\
\kappa_{\alpha \beta \gamma} & =\sum_{k=1}^{4}\left(a_{k}+b_{k} x_{3}+\frac{1}{2} c_{k} x_{3}^{2}\right) \kappa_{\alpha \beta \gamma}^{(k)}+\eta_{\alpha \beta \gamma}+x_{3} \zeta_{\alpha \beta \gamma}
\end{aligned}
$$




$$
\begin{aligned}
\kappa_{\alpha \beta 3} & =\sum_{k=1}^{4}\left(a_{k}+b_{k} x_{3}+\frac{1}{2} c_{k} x_{3}^{2}\right) \kappa_{\alpha \beta 3}^{(k)}+\eta_{a \beta 3}+x_{3} \zeta_{\alpha \beta 3}, \\
\kappa_{\beta 3 \alpha} & =\varepsilon_{3 \beta \alpha}\left(a_{4}+b_{4} x_{3}+\frac{1}{2} c_{4} x_{3}^{2}\right)+\sum_{k=1}^{4}\left(b_{k}+c_{k} x_{3}\right) u_{\alpha, \beta}^{(k)}+v_{\alpha, \beta}, \\
\kappa_{\alpha 33} & =a_{\alpha}+b_{\alpha} x_{3}+\frac{1}{2} c_{\alpha} x_{3}^{2}+\frac{1}{2} \sum_{k=1}^{4}\left(b_{k}+c_{k} x_{3}\right) e_{\alpha 3}^{(k)}+\frac{1}{2} \xi_{\alpha 3}, \\
\kappa_{33 \alpha} & =-a_{\alpha}-b_{\alpha} x_{3}-\frac{1}{2} c_{\alpha} x_{3}^{2}+\varepsilon_{3 \beta \alpha}\left(b_{4}+c_{4} x_{3}\right) x_{\beta}+\sum_{k=1}^{4} c_{k} u_{\alpha}^{(k)}, \\
\kappa_{333} & =b_{1} x_{1}+b_{2} x_{2}+b_{3}+\left(c_{1} x_{1}+c_{2} x_{2}+c_{3}\right) x_{3}+\sum_{k=1}^{4} c_{k} u_{3}^{(k)} .
\end{aligned}
$$

We introduce the notations

$$
\begin{aligned}
t_{\alpha \beta} & =\lambda \gamma_{\rho \rho} \delta_{\alpha \beta}+2 \mu \gamma_{\alpha \beta}+f\left(\varepsilon_{\alpha \rho 3} \eta_{\beta \rho 3}+\varepsilon_{\beta \rho 3} \eta_{\alpha \rho 3}\right) \\
t_{\alpha 3} & =2 \mu \gamma_{\alpha 3}+f \varepsilon_{\rho \beta 3} \eta_{\alpha \rho \beta}, \quad t_{33}=\lambda \gamma_{\rho \rho}, \\
\nu_{\alpha \beta \gamma} & =\frac{1}{2} \alpha_{1}\left(\eta_{\rho \rho \alpha} \delta_{\beta \gamma}+2 \eta_{\gamma \rho \rho} \delta_{\alpha \beta}+\eta_{\rho \rho \beta} \delta_{\alpha \gamma}\right)+ \\
& +\alpha_{2}\left(\eta_{\alpha \rho \rho} \delta_{\beta \gamma}+\eta_{\beta \rho \rho} \delta_{\alpha \gamma}\right)+2 \alpha_{3} \eta_{\rho \rho \gamma} \delta_{\alpha \beta}+2 \alpha_{4} \eta_{\alpha \beta \gamma}+ \\
& +\alpha_{5}\left(\eta_{\gamma \beta \alpha}+\eta_{\gamma \alpha \beta}\right)+f\left(\varepsilon_{\alpha \gamma 3} \gamma_{\beta 3}+\varepsilon_{\beta \gamma 3} \gamma_{\alpha 3}\right), \\
\nu_{\alpha \beta 3} & =2 \alpha_{3} \eta_{\rho \rho 3} \delta_{\alpha \beta}+2 \alpha_{4} \eta_{\alpha \beta 3}+f\left(\varepsilon_{\rho \alpha 3} \gamma_{\beta \rho}+\varepsilon_{\rho \beta 3} \gamma_{\alpha \rho}\right), \\
\nu_{\alpha 33} & =\frac{1}{2} \alpha_{1} \eta_{\rho \rho \alpha}+\alpha_{2} \eta_{\alpha \rho \rho}+f \varepsilon_{3 \rho \alpha} \gamma_{3 \rho}, \\
\nu_{33 \alpha} & =\alpha_{1} \eta_{\alpha \rho \rho}+2 \alpha_{3} \eta_{\rho \rho \alpha}+2 f \varepsilon_{3 \alpha \rho} \gamma_{3 \rho}, \\
\nu_{\alpha 3 \beta} & =\frac{1}{2} \alpha_{1} \eta_{\rho \rho 3}+\alpha_{5} \eta_{\beta \alpha 3}+f \varepsilon_{3 \beta \rho} \gamma_{\alpha \rho}, \quad \nu_{333}=\left(\alpha_{1}+2 \alpha_{3}\right) \eta_{\rho \rho 3} .
\end{aligned}
$$

Clearly, $t_{i j}$ and $\nu_{i j k}$ are the stress and the double stress tensors in the generalized plane strain characterized by the displacements $w_{k}$. The stress tensor and the double stress tensor corresponding to the strain measures $\xi_{i j}$ and $\zeta_{i j k}$ are defined by

$$
\begin{aligned}
s_{\alpha \beta} & =\lambda \xi_{\rho \rho} \delta_{\alpha \beta}+2 \mu \xi_{\alpha \beta}+f\left(\varepsilon_{\alpha \rho 3} \zeta_{\beta \rho 3}+\varepsilon_{\beta \rho 3} \zeta_{\alpha \rho 3}\right), \\
s_{\alpha 3} & =2 \mu \xi_{\alpha 3}+f \varepsilon_{\rho \beta 3} \zeta_{\alpha \rho 3}, s_{33}=\lambda \xi_{\rho \rho}, \\
m_{\alpha \beta \gamma} & =\frac{1}{2} \alpha_{1}\left(\zeta_{\rho \rho \alpha} \delta_{\beta \gamma}+2 \zeta_{\gamma \rho \rho} \delta_{\alpha \beta}+\zeta_{\rho \rho \beta} \delta_{\alpha \gamma}\right)+ \\
& +\alpha_{2}\left(\zeta_{\alpha \rho \rho} \delta_{\beta \gamma}+\zeta_{\beta \rho \rho} \delta_{\alpha \gamma}\right)+2 \alpha_{3} \zeta_{\rho \rho \gamma} \delta_{\alpha \beta}+2 \alpha_{4} \zeta_{\alpha \beta \gamma}+ \\
& +\alpha_{5}\left(\zeta_{\gamma \beta \alpha}+\zeta_{\gamma \alpha \beta}+f\left(\varepsilon_{\alpha \gamma 3} \xi_{\beta 3}+\varepsilon_{\beta \gamma 3} \xi_{\alpha 3}\right),\right. \\
m_{\alpha \beta 3} & =2 \alpha_{3} \zeta_{\rho \rho 3} \delta_{\alpha \beta}+2 \alpha_{4} \zeta_{\alpha \beta 3}+f\left(\varepsilon_{\rho \alpha 3} \xi_{\beta \rho}+\varepsilon_{\rho \beta 3} \xi_{\alpha \rho}\right), \\
m_{\alpha 33} & =\frac{1}{2} \alpha_{1} \zeta_{\rho \rho \alpha}+\alpha_{2} \zeta_{\alpha \rho \rho}+f \varepsilon_{3 \rho \alpha} \xi_{3 \rho}, \\
m_{33 \alpha} & =\alpha_{1} \zeta_{\alpha \rho \rho}+\alpha_{2} \zeta_{\rho \rho \alpha}+2 f \varepsilon_{3 \alpha \rho} \xi_{3 \rho}, \\
m_{\alpha 3 \beta} & =\frac{1}{2} \alpha_{1} \zeta_{\rho \rho 3}+\alpha_{5} \zeta_{\beta \alpha 3}+f \varepsilon_{3 \beta \rho} \xi_{\alpha \rho}, m_{333}=\left(\alpha_{1}+2 \alpha_{3}\right) \zeta_{\rho \rho 3} .
\end{aligned}
$$


It follows from the constitutive equations (2) and the relations (32)-(36) that the stress tensor $\tau_{i j}$ is given by

$$
\begin{aligned}
\tau_{\alpha \beta} & =t_{\alpha \beta}+x_{3} s_{\alpha \beta}+\left\{\lambda \left[a_{1} x_{1}+a_{2} x_{2}+a_{3}+\left(b_{1} x_{1}+b_{2} x_{2}+b_{3}\right) x_{3}+\right.\right. \\
& \left.\left.+\frac{1}{2}\left(c_{1} x_{1}+c_{2} x_{2}+c_{3}\right) x_{3}^{2}\right]-2 f\left(a_{4}+b_{4} x_{3}+\frac{1}{2} c_{4} x_{3}^{2}\right)\right\} \delta_{\alpha \beta}+ \\
& +\sum_{k=1}^{4}\left(a_{k}+b_{k} x_{3}+\frac{1}{2} c_{k} x_{3}^{2}\right) \tau_{\alpha \beta}^{(k)}+T_{\alpha \beta}+x_{3} S_{\alpha \beta}, \\
\tau_{\alpha 3} & =t_{\alpha 3}+x_{3} s_{\alpha 3}+2 f \varepsilon_{\alpha \rho 3}\left(a_{\rho}+b_{\rho} x_{3}+\frac{1}{2} c_{\rho} x_{3}^{2}\right)+ \\
& +\mu \varepsilon_{3 \beta \alpha}\left(a_{4}+b_{4} x_{3}+\frac{1}{2} c_{4} x_{3}^{2}\right) x_{\beta}+ \\
& +\sum_{k=1}^{4}\left(a_{k}+b_{k} x_{3}+\frac{1}{2} c_{k} x_{3}^{2}\right) \tau_{\alpha 3}^{(k)}+T_{\alpha 3}+x_{3} S_{\alpha 3}, \\
\tau_{33} & =t_{33}+x_{3} s_{33}+(\lambda+2 \mu)\left[a_{1} x_{1}+a_{2} x_{2}+a_{3}+\left(b_{1} x_{1}+b_{2} x_{2}+b_{3}\right) x_{3}+\right. \\
& \left.+\frac{1}{2}\left(c_{1} x_{1}+c_{2} x_{2}+c_{3}\right) x_{3}^{2}\right]+4 f\left(a_{4}+b_{4} x_{3}+\frac{1}{2} c_{4} x_{3}^{2}\right)+ \\
& +\lambda \sum_{k=1}^{4}\left(a_{k}+b_{k} x_{3}+\frac{1}{2} c_{k} x_{3}^{2}\right) u_{\rho, \rho}^{(k)}+T_{33}+x_{3} S_{33},
\end{aligned}
$$

where

$$
\begin{aligned}
& T_{\alpha \beta}=f\left[\varepsilon_{3 \rho \alpha} v_{\rho, \beta}+\varepsilon_{3 \rho \beta} v_{\rho, \alpha}+\sum_{k=1}^{4} b_{k}\left(\varepsilon_{3 \rho \alpha} u_{\rho, \beta}^{(k)}+\varepsilon_{3 \rho \beta} u_{\rho, \alpha}^{(k)}\right)\right], \\
& S_{\alpha \beta}=f \sum_{k=1}^{4} c_{k}\left(\varepsilon_{3 \rho \alpha} u_{\rho, \beta}^{(k)}+\varepsilon_{3 \rho \beta} u_{\rho, \alpha}^{(k)}\right), \\
& T_{\alpha 3}=\mu\left(v_{\alpha}+\sum_{k=1}^{4} b_{k} u_{\alpha}^{(k)}\right)+f \varepsilon_{\alpha \rho 3}\left[v_{3, \rho}+\sum_{k=1}^{4}\left(b_{k} u_{3, \rho}^{(k)}-c_{k} u_{\rho}^{(k)}\right)\right]-f b_{4} x_{\alpha}, \\
& S_{\alpha 3}=\mu \sum_{k=1}^{4} c_{k} u_{\alpha}^{(k)}+f \varepsilon_{\alpha \rho 3} \sum_{k=1}^{4} c_{k} u_{3, \rho}^{(k)}-f c_{4} x_{\alpha}, \\
& T_{33}=(\lambda+2 \mu)\left(v_{3}+\sum_{k=1}^{4} b_{k} u_{3}^{(k)}\right)+2 f \varepsilon_{3 \alpha \beta}\left(v_{\alpha, \beta}+\sum_{k=1}^{4} b_{j} u_{\alpha, \beta}^{(k)}\right), \\
& S_{33}=(\lambda+2 \mu) \sum_{k=1}^{4} c_{k} u_{3}^{(k)}+2 f \varepsilon_{3 \alpha \beta} \sum_{k=1}^{4} c_{k} u_{\alpha, \beta}^{(k)} .
\end{aligned}
$$

The double stress tensor has the following components

$$
\begin{aligned}
\mu_{111} & =\nu_{111}+x_{3} m_{111}+2\left(\alpha_{2}-\alpha_{3}\right)\left(a_{1}+b_{1} x_{3}+\frac{1}{2} c_{1} x_{3}^{2}\right)+ \\
& +\sum_{k=1}^{4}\left(a_{k}+b_{k} x_{3}+\frac{1}{2} c_{k} x_{3}^{2}\right) \mu_{111}^{(k)}+N_{111}+x_{3} M_{111} \\
\mu_{222} & =\nu_{222}+x_{3} m_{222}+2\left(\alpha_{2}-\alpha_{3}\right)\left(a_{2}+b_{2} x_{3}+\frac{1}{2} c_{2} x_{3}^{2}\right)+
\end{aligned}
$$




$$
\begin{aligned}
& +\sum_{k=1}^{4}\left(a_{k}+b_{k} x_{3}+\frac{1}{2} c_{k} x_{3}^{2}\right) \mu_{222}^{(k)} N_{222}+x_{3} M_{222} \\
& \mu_{221}=\nu_{221}+x_{3} m_{221}+\left(\alpha_{1}-2 \alpha_{3}\right)\left(a_{1}+b_{1} x_{3}+\frac{1}{2} c_{1} x_{3}^{2}\right)- \\
& -f\left(a_{4}+b_{4} x_{3}+\frac{1}{2} c_{4} x_{3}^{2}\right) x_{1}+ \\
& +\sum_{k=1}^{4}\left(a_{k}+b_{k} x_{3}+\frac{1}{2} c_{k} x_{3}^{2}\right) \mu_{221}^{(k)}+N_{221}+x_{3} M_{221}, \\
& \mu_{112}=\nu_{112}+x_{3} m_{112}+\left(\alpha_{1}-2 \alpha_{3}\right)\left(a_{2}+b_{2} x_{3}+\frac{1}{2} c_{2} x_{3}^{2}\right)- \\
& -f\left(a_{4}+b_{4} x_{3}+\frac{1}{2} c_{4} x_{3}^{2}\right) x_{2}+ \\
& +\sum_{k=1}^{4}\left(a_{k}+b_{k} x_{3}+\frac{1}{2} c_{k} x_{3}^{2}\right) \mu_{112}^{(k)}+N_{112}+x_{3} M_{112}, \\
& \mu_{121}=\nu_{121}+x_{3} m_{121}+\frac{1}{2}\left(2 \alpha_{2}-\alpha_{1}\right)\left(a_{2}+b_{2} x_{3}+\frac{1}{2} c_{2} x_{3}^{2}\right)+ \\
& +\frac{1}{2} f\left(a_{4}+b_{4} x_{3}+\frac{1}{2} c_{4} x_{3}^{2}\right) x_{2}+ \\
& +\sum_{k=1}^{4}\left(a_{k}+b_{k} x_{3}+\frac{1}{2} c_{k} x_{3}^{2}\right) \mu_{121}^{(k)}+N_{121}+x_{3} M_{121} \text {, } \\
& \mu_{122}=\nu_{122}+x_{3} m_{122}+\frac{1}{2}\left(2 \alpha_{2}-\alpha_{1}\right)\left(a_{1}+b_{1} x_{3}+\frac{1}{2} c_{1} x_{3}^{2}\right)+ \\
& +\frac{1}{2} f\left(a_{4}+b_{4} x_{3}+\frac{1}{2} c_{4} x_{3}^{2}\right) x_{1}+ \\
& +\sum_{k=1}^{4}\left(a_{k}+b_{k} x_{3}+\frac{1}{2} c_{k} x_{3}^{2}\right) \mu_{122}^{(k)}+N_{122}+x_{3} M_{122}, \\
& \mu_{\rho 33}=\nu_{\rho 33}+x_{3} m_{\rho 33}+\frac{1}{2}\left(2 \alpha_{2}-\alpha_{1}+4 \alpha_{4}\right)\left(a_{\rho}+b_{\rho} x_{3}+\frac{1}{2} c_{\rho} x_{3}^{2}\right)- \\
& -\frac{1}{2} f\left(a_{4}+b_{4} x_{3}+\frac{1}{2} c_{4} x_{3}^{2}\right) x_{\rho}+ \\
& +\sum_{k=1}^{4}\left(a_{k}+b_{k} x_{3}+\frac{1}{2} c_{k} x_{3}^{2}\right) \mu_{\rho 33}^{(k)}+N_{\rho 33}+x_{3} M_{\rho 33} \text {, } \\
& \mu_{33 \rho}=\nu_{33 \rho}+x_{3} m_{33 \rho}+\left(\alpha_{1}-2 \alpha_{3}-2 \alpha_{4}+2 \alpha_{5}\right)\left(a_{\rho}+b_{\rho} x_{3}+\frac{1}{2} c_{\rho} x_{3}^{2}\right)+ \\
& +f\left(a_{4}+b_{4} x_{3}+\frac{1}{2} c_{4} x_{3}^{2}\right) x_{\rho}+ \\
& +\sum_{k=1}^{4}\left(a_{k}+b_{k} x_{3}+\frac{1}{2} c_{k} x_{3}^{2}\right) \mu_{33 \rho}^{(k)}+N_{33 \rho}+x_{3} M_{33 \rho}, \\
& \mu_{\alpha 3 \beta}=\nu_{\alpha 3 \beta}+x_{3} m_{\alpha 3 \beta}+\left(2 \alpha_{4}-\alpha_{5}\right)\left(a_{4}+b_{4} x_{3}+\frac{1}{2} c_{4} x_{3}^{2}\right) \varepsilon_{\alpha \beta 3}+ \\
& +f\left[a_{1} x_{1}+a_{2} x_{2}+a_{3}+\left(b_{1} x_{1}+b_{2} x_{2}+b_{3}\right) x_{3}+\right. \\
& \left.+\frac{1}{2}\left(c_{1} x_{1}+c_{2} x_{2}+c_{3}\right) x_{3}^{2}\right] \varepsilon_{\alpha \beta 3}+
\end{aligned}
$$




$$
\begin{aligned}
& +\sum_{k=1}^{4}\left(a_{k}+b_{k} x_{3}+\frac{1}{2} c_{j} x_{3}^{2}\right) \mu_{\alpha 3 \beta}^{(k)}+N_{\alpha 3 \beta}+x_{3} M_{\alpha 3 \beta}, \\
\mu_{\alpha \beta 3}= & \nu_{\alpha \beta 3}+x_{3} m_{\alpha \beta 3}+\sum_{k=1}^{4}\left(a_{k}+b_{k} x_{3}+\right. \\
& \left.+\frac{1}{2} c_{k} x_{3}^{2}\right) \mu_{\alpha \beta 3}^{(k)}+N_{a \beta 3}+x_{3} M_{\alpha \beta 3}, \\
\mu_{333}= & \nu_{333}+x_{3} m_{333}+\left(\alpha_{1}+2 \alpha_{3}\right) \sum_{k=1}^{4}\left(a_{k}+b_{k} x_{3}+\frac{1}{2} c_{k} x_{3}^{2}\right) \kappa_{333}^{(k)}+N_{333}+x_{3} M_{333},
\end{aligned}
$$

where we have used the notations

$$
\begin{aligned}
N_{111} & =\left(\alpha_{1}+2 \alpha_{3}\right)\left(\sum_{k=1}^{4} c_{k} u_{1}^{(k)}-b_{4} x_{2}\right)+\left(\alpha_{1}+2 \alpha_{2}\right)\left(v_{3,1}+\sum_{k=1}^{4} b_{k} u_{3,1}^{(k)}\right), \\
M_{111} & =-\left(\alpha_{1}+2 \alpha_{3}\right) c_{4} x_{2}+\left(\alpha_{1}+2 \alpha_{2}\right) \sum_{k=1}^{4} c_{k} u_{3,1}^{(k)}, \\
N_{222} & =\left(\alpha_{1}+2 \alpha_{3}\right)\left(b_{4} x_{1}+\sum_{k=1}^{4} c_{k} u_{2}^{(k)}\right)+\left(\alpha_{1}+2 \alpha_{2}\right)\left(v_{3,2}+\sum_{k=1}^{4} b_{k} u_{3,2}^{(k)}\right), \\
M_{222} & =\left(\alpha_{1}+2 \alpha_{3}\right) c_{4} x_{1}+\left(\alpha_{1}+2 \alpha_{2}\right) \sum_{k=1}^{4} c_{k} u_{3,2}^{(k)}, \\
N_{221} & =2 \alpha_{3}\left(\sum_{k=1}^{4} c_{k} u_{1}^{(k)}-b_{4} x_{2}\right)+\alpha_{1}\left(v_{3,1}+\sum_{k=1}^{4} b_{k} u_{3,1}^{(k)}\right)-f\left(v_{2}+\sum_{k=1}^{4} b_{k} u_{2}^{(k)}\right), \\
M_{221} & =\alpha_{1} \sum_{k=1}^{4} c_{k} u_{3,1}^{(k)}-2 \alpha_{3} c_{4} x_{2}-f \sum_{k=1}^{4} c_{k} u_{2}^{(k)}, \\
N_{112} & =\alpha_{1}\left(v_{3,2}+\sum_{k=1}^{4} b_{k} u_{3,2}^{(k)}\right)+2 \alpha_{3}\left(b_{4} x_{1}+\sum_{k=1}^{4} b_{k} u_{1}^{(k)}\right)+ \\
& +f\left(v_{1}+\sum_{k=1}^{4} b_{k} u_{1}^{(k)}\right), \\
M_{112} & =\alpha_{1} \sum_{k=1}^{4} c_{k} u_{3,2}^{(k)}+2 \alpha_{3} c_{4} x_{1}+f \sum_{k=1}^{4} c_{k} u_{1}^{(k)}, \\
N_{121} & =\frac{1}{2} \alpha_{1}\left(b_{4} x_{1}+\sum_{k=1}^{4} c_{k} u_{2}^{(k)}\right)+\alpha_{2}\left(v_{3,2}+\sum_{k=1}^{4} b_{k} u_{3,2}^{(k)}\right)- \\
& -\frac{1}{2} f\left(v_{1}+\sum_{k=1}^{4} b_{k} u_{1}^{(k)}\right), \\
M_{121} & =\frac{1}{2} \alpha_{1} c_{4} x_{1}+\alpha_{2} \sum_{k=1}^{4} c_{k} u_{3,2}^{(k)}-\frac{1}{2} f \sum_{k=1}^{4} c_{k} u_{1}^{(k)} \\
N_{122} & =\frac{1}{2} \alpha_{1}\left(\sum_{k=1}^{4} c_{k} u_{1}^{(k)}-b_{4} x_{2}\right)+\alpha_{2}\left(v_{3,1}+\sum_{k=1}^{4} b_{k} u_{3,1}^{(k)}\right)+
\end{aligned}
$$




$$
\begin{aligned}
& +\frac{1}{2} f\left(v_{2}+\sum_{k=1}^{4} b_{k} u_{2}^{(k)}\right) \\
& M_{122}=-\frac{1}{2} \alpha_{1} c_{4} x_{2}+\alpha_{2} \sum_{k=1}^{4} c_{k} u_{3,1}^{(k)}+\frac{1}{2} f \sum_{k=1}^{4} c_{k} u_{2}^{(k)} \\
& N_{\rho 33}=\frac{1}{2}\left(\alpha_{1}+2 \alpha_{5}\right)\left(\sum_{k=1}^{4} c_{k} u_{\rho}^{(k)}-\varepsilon_{3 \rho \beta} b_{4} x_{\beta}\right)+ \\
& +\left(\alpha_{2}+2 \alpha_{4}+\alpha_{5}\right)\left(v_{3, \rho}+\sum_{k=1}^{4} b_{k} u_{3, \rho}^{(k)}\right)+\frac{1}{2} f \varepsilon_{3 \rho \beta}\left(v_{\beta}+\sum_{k=1}^{4} b_{k} u_{\beta}^{(k)}\right), \\
& M_{\rho 33}=-\frac{1}{2}\left(\alpha_{1}+2 \alpha_{5}\right) \varepsilon_{3 \rho \beta} c_{4} x_{\beta}+\left(\alpha_{2}+2 \alpha_{4}+\alpha_{5}\right) \sum_{k=1}^{4} c_{k} u_{3, \rho}^{(k)}+ \\
& +\frac{1}{2} f \varepsilon_{3 \rho \beta} \sum_{k=1}^{4} c_{k} u_{\beta}^{(k)} \\
& N_{33 \rho}=2\left(\alpha_{3}+\alpha_{4}\right)\left(\sum_{k=1}^{4} c_{k} u_{\rho}^{(k)}-\varepsilon_{3 \rho \beta} b_{4} x_{\beta}\right)+\left(\alpha_{1}+2 \alpha_{5}\right)\left(v_{3, \rho}+\sum_{k=1}^{4} b_{k} u_{3, \rho}^{(k)}\right)+ \\
& +f \varepsilon_{3 \rho \beta}\left(v_{\beta}+\sum_{k=1}^{4} b_{k} u_{\beta}^{(k)}\right) \\
& M_{33 \rho}=-2\left(\alpha_{3}+\alpha_{4}\right) \varepsilon_{3 \rho \beta} c_{4} x_{\beta}+\left(\alpha_{1}+2 \alpha_{5}\right) \sum_{k=1}^{4} c_{k} u_{3, \rho}^{(k)}+ \\
& +f \varepsilon_{3 \rho \beta} \sum_{k=1}^{4} c_{k} u_{\beta}^{(k)} \\
& N_{\alpha 3 \beta}=2 \alpha_{4}\left(v_{\beta, \alpha}+\sum_{k=1}^{4} b_{k} u_{\beta, \alpha}^{(k)}\right)+\alpha_{5}\left(v_{\alpha, \beta}+\sum_{k=1}^{4} b_{k} u_{\alpha, \beta}^{(k)}\right)+ \\
& +\delta_{a \beta}\left[\frac{1}{2}\left(\alpha_{1}+2 \alpha_{2}\right)\left(b_{1} x_{1}+b_{2} x_{2}+b_{3}+\sum_{k=1}^{4} c_{k} u_{3}^{(k)}\right)+\right. \\
& \left.+\alpha_{2}\left(v_{\rho, \rho}+\sum_{k=1}^{4} b_{k} u_{\rho, \rho}^{(k)}\right)\right]+f\left(v_{3}+\sum_{k=1}^{4} b_{k} u_{3}^{(k)}\right) \varepsilon_{\alpha \beta 3} \\
& M_{\alpha 3 \beta}=\sum_{k=1}^{4} c_{k}\left[2 \alpha_{4} u_{\beta, \alpha}^{(k)}+\alpha_{5} u_{\alpha, \beta}^{(k)}+\alpha_{2} \delta_{\alpha \beta} u_{\rho, \rho}^{(k)}+\right. \\
& \left.+f \varepsilon_{\alpha \beta 3} u_{3}^{(k)}\right]+\frac{1}{2}\left(\alpha_{1}+2 \alpha_{2}\right) \delta_{\alpha \beta}\left(c_{1} x_{1}+c_{2} x_{2}+c_{3}\right), \\
& N_{\alpha \beta 3}=\delta_{\alpha \beta}\left[\left(\alpha_{1}+2 \alpha_{3}\right)\left(b_{1} x_{1}+b_{2} x_{2}+b_{3}+\sum_{k=1}^{4} c_{k} u_{3}^{(k)}\right)+\right. \\
& \left.+\alpha_{1}\left(v_{\rho, \rho}+\sum_{k=1}^{4} b_{k} u_{\rho, \rho}^{(k)}\right)\right]+2 \alpha_{5}\left(\xi_{\alpha \beta}+\sum_{k=1}^{4} b_{k} e_{\alpha \beta}^{(k)}\right), \\
& M_{\alpha \beta 3}=\left(\alpha_{1}+2 \alpha_{3}\right) \delta_{\alpha \beta}\left(c_{1} x_{1}+c_{2} x_{2}+c_{3}\right)+ \\
& +\sum_{k=1}^{4} c_{k}\left[\left(\alpha_{1}+2 \alpha_{3}\right) \delta_{a \beta} u_{3}^{(k)}+\alpha_{1} \delta_{\alpha \beta} u_{\rho, \rho}^{(k)}+2 \alpha_{5} e_{\alpha \beta}^{(k)}\right],
\end{aligned}
$$




$$
\begin{aligned}
N_{333} & =2\left(\alpha_{1}+\alpha_{2}+\alpha_{3}+\alpha_{4}+\alpha_{5}\right)\left(b_{1} x_{1}+b_{2} x_{2}+b_{3}+\sum_{k=1}^{4} c_{k} u_{3}^{(k)}\right)+ \\
& +\left(\alpha_{1}+2 \alpha_{2}\right)\left(v_{\rho, \rho}+\sum_{k=1}^{4} c_{k} u_{\rho, \rho}^{(k)}\right) \\
M_{333} & =2\left(\alpha_{1}+\alpha_{2}+\alpha_{3}+\alpha_{4}+\alpha_{5}\right)\left(c_{1} x_{1}+c_{2} x_{2}+c_{3}\right)+\left(\alpha_{1}+2 \alpha_{2}\right) \sum_{k=1}^{4} c_{k} u_{\rho, \rho}^{(k)} .
\end{aligned}
$$

We introduce the functions $G_{j}^{(0)}$ and $G_{j}^{(1)}$ by

$$
\begin{aligned}
G_{\alpha}^{(0)} & =F_{\alpha}+T_{\beta \alpha, \beta}-N_{\rho \eta \alpha, \rho \eta}-2 M_{3 \rho \alpha, \rho}+S_{\alpha 3} \\
& -\sum_{k=1}^{4}\left(2 b_{k} \mu_{3 \rho \alpha, \rho}^{(k)}-b_{k} \tau_{\alpha 3}^{(k)}+c_{k} \mu_{33 \alpha}^{(k)}\right) \\
& +4 f \varepsilon_{3 \alpha \beta} b_{\beta}+\mu \varepsilon_{3 \rho \alpha} x_{\rho} b_{4}-\left(\alpha_{1}-2 \alpha_{3}-2 \alpha_{4}+2 \alpha_{5}\right) c_{\alpha} \\
& -f c_{4} x_{\alpha}+s_{\alpha 3}-2 m_{3 \rho \alpha, \rho}, \\
G_{3}^{(0)} & =F_{3}+T_{\beta 3, \beta}-N_{\rho \eta 3, \rho \eta}-2 M_{\rho 33, \rho}+S_{33} \\
& +\sum_{k=1}^{4}\left[\lambda b_{k} u_{\rho, \rho}^{(k)}-2 b_{k} \mu_{\rho 33, \rho}^{(k)}-\left(\alpha_{1}+2 \alpha_{3}\right) c_{k} \kappa_{333}^{(k)}\right] \\
& +(\lambda+2 \mu)\left(b_{1} x_{1}+b_{2} x_{2}+b_{3}\right)+6 f b_{4}+s_{33}-2 m_{\rho 33, \rho}
\end{aligned}
$$

and

$$
\begin{aligned}
G_{\alpha}^{(1)} & =S_{\beta \alpha, \beta}-M_{\rho \eta \alpha, \rho \eta}+4 f \varepsilon_{\alpha \rho 3} c_{\rho}-\mu \varepsilon_{\alpha \rho 3} c_{4} x_{\rho}+ \\
& +\sum_{k=1}^{4} c_{k}\left(\tau_{\alpha 3}^{(k)}-2 \mu_{\rho 3 \alpha, \rho}^{(k)}\right), \\
G_{3}^{(1)} & =S_{\rho 3, \rho}-M_{\rho \eta 3, \rho \eta}+(\lambda+2 \mu)\left(c_{1} x_{1}+c_{2} x_{2}+c_{3}\right)+6 f c_{4}+ \\
& +\sum_{k=1}^{4} c_{k}\left(\lambda u_{\rho, \rho}^{(k)}-2 \mu_{\rho 33, \rho}^{(k)}\right) .
\end{aligned}
$$

With the help of relations (29), (37), (39), (41) and (42) we find that the equilibrium equations (3) reduce to the following equations

$$
t_{\beta j, \beta}-\nu_{\rho \eta j, \rho \eta}+G_{j}^{(0)}=0 \text { on } \Sigma
$$

and

$$
s_{\beta j, \beta}-m_{\rho \eta j, \rho \eta}+G_{j}^{(1)}=0 \text { on } \Sigma .
$$

Let us introduce the notations

$$
\begin{aligned}
& \Pi_{j}^{(0)}=\left(t_{\beta j}-\nu_{\rho \beta j, \rho}\right) n_{\beta}-D_{\eta}\left(n_{\rho} \nu_{\rho \eta j}\right)+\left(D_{\nu} n_{\nu}\right) n_{\rho} n_{\eta} \nu_{\rho \eta j}, \\
& \Pi_{j}^{(1)}=\left(s_{\beta j}-m_{\rho \beta j, \rho}\right) n_{\beta}-D_{\eta}\left(n_{\rho} m_{\rho \eta j}\right)+\left(D_{\nu} n_{\nu}\right) n_{\rho} n_{\eta} m_{\rho \eta j}, \\
& \Lambda_{j}^{(0)}=\nu_{\rho \eta j} n_{\rho} n_{\eta}, \quad \Lambda_{j}^{(1)}=m_{\rho \eta j} n_{\rho} n_{\eta}
\end{aligned}
$$


and

$$
\begin{aligned}
H_{j}^{(0)} & =\left(T_{\beta j}-N_{\rho \beta j, \rho}\right) n_{\beta}-D_{\eta}\left(n_{\rho} N_{\rho \eta j}\right)+\left(D_{\nu} n_{\nu}\right) n_{\rho} n_{\eta} N_{\rho \eta j}, \\
H_{j}^{(1)} & =\left(S_{\beta j}-M_{\rho \beta j, \rho}\right) n_{\beta}-D_{\eta}\left(n_{\rho} M_{\rho \eta j}\right)+\left(D_{\nu} n_{\nu}\right) n_{\rho} n_{\eta} M_{\rho \eta j}, \\
L_{j}^{(0)} & =N_{\rho \eta j} n_{\rho} n_{\eta}, \quad L_{j}^{(1)}=M_{\rho \eta j} n_{\rho} n_{\eta} .
\end{aligned}
$$

If we take into account the relations (30), (37) and (39) then we see that the conditions on the lateral surface (8) reduce to

$$
\Pi_{j}^{(0)}=\Phi_{j}^{(0)}, \Lambda_{j}^{(0)}=\Psi_{j}^{(0)} \text { on } \Gamma_{1}
$$

and

$$
\Pi_{j}^{(1)}=\Phi_{j}^{(1)}, \Lambda_{j}^{(1)}=\Psi_{j}^{(1)} \text { on } \Gamma_{1},
$$

where we have used the notations

$$
\begin{aligned}
\Phi_{\alpha}^{(0)} & =\widetilde{P}_{\alpha}+2 n_{\rho}\left(m_{3 \rho \alpha}+M_{3 \rho \alpha}+\sum_{k=1}^{4} b_{k} \mu_{3 \rho \alpha}^{(k)}\right)- \\
& -2 \varepsilon_{\alpha \rho 3} n_{\rho}\left[\left(2 \alpha_{4}-\alpha_{5}\right) b_{4}+f\left(b_{1} x_{1}+b_{2} x_{2}+b_{3}\right)\right]-H_{\alpha}^{(0)}, \\
\Phi_{3}^{(0)} & =\widetilde{P}_{3}+2 n_{\rho}\left[m_{\rho 33}+M_{\rho 33}+\frac{1}{2}\left(2 \alpha_{2}-\alpha_{1}+4 \alpha_{4}\right) b_{\rho}-\frac{1}{2} f b_{4} x_{\rho}+\right. \\
& \left.+\sum_{k=1}^{4} b_{k} \mu_{\rho 33}^{(k)}\right]-H_{3}^{(0)}, \\
\Phi_{\alpha}^{(1)} & =2 n_{\rho}\left\{\varepsilon_{\rho \alpha 3}\left[\left(2 \alpha_{4}-\alpha_{5}\right) c_{4}+f\left(c_{1} x_{1}+c_{2} x_{2}+c_{3}\right)\right]+\right. \\
& \left.+\sum_{k=1}^{4} c_{k} \mu_{\rho 3 \alpha}^{(k)}\right\}-H_{\alpha}^{(1)}, \\
\Phi_{3}^{(1)} & =2 n_{\rho}\left[\frac{1}{2}\left(2 \alpha_{2}-\alpha_{1}+4 \alpha_{4}\right) c_{\rho}-\frac{1}{2} f c_{4} x_{\rho}+\right. \\
& \left.+\sum_{k=1}^{4} c_{k} \mu_{\rho 33}^{(k)}\right]-H_{3}^{(1)}, \\
\Psi_{j}^{(0)} & =\widetilde{R}_{j}-L_{j}^{(0)}, \quad \Psi_{j}^{(1)}=-L_{j}^{(1)} .
\end{aligned}
$$

Let us denote by $\left(\mathcal{A}_{0}\right)$ the generalized plane strain problem defined by the geometrical equations (32), the constitutive equations (35), the equilibrium equations (43) and the boundary conditions (47). We denote by $\left(\mathcal{A}_{1}\right)$ the generalized plane strain problem characterized by the equations (33), (36), (44) on $\Sigma$, and the boundary conditions (48). The necessary and sufficient conditions to solve the problem $\left(\mathcal{A}_{1}\right)$ are

$$
\int_{\Sigma_{1}} G_{j}^{(1)} d a+\int_{\Gamma_{1}} \Phi_{j}^{(1)} d s=0, \int_{\Sigma_{1}} \varepsilon_{3 \alpha \beta} x_{\alpha} G_{\beta}^{(1)} d a+\int_{\Gamma_{1}} \varepsilon_{3 \alpha \beta}\left(x_{\alpha} \Phi_{\beta}+n_{\alpha} \Psi_{\beta}^{(1)}\right) d s=0
$$


By using the divergence theorem we find that

$$
\begin{aligned}
& \int_{\Sigma_{1}}\left(S_{\beta j, \beta}-M_{\rho \eta j, \rho \eta}\right) d a+\int_{\Gamma} H_{j}^{(1)} d s=0, \\
& \int_{\Sigma_{1}} \varepsilon_{3 \alpha \beta} x_{\alpha}\left(S_{\rho \beta, \rho}-M_{\nu \eta \beta, \nu \eta}\right) d a+\int_{\Gamma_{1}} \varepsilon_{3 \alpha \beta}\left(x_{\alpha} H_{\beta}^{(1)}+n_{\alpha} L_{\beta}^{(1)}\right) d s=0 .
\end{aligned}
$$

In view of (37), (39), (42) and (49) we obtain

$$
\int_{\Sigma_{1}} G_{\alpha}^{(1)} d a+\int_{\Gamma_{1}} \Phi_{\alpha}^{(1)} d s=\int_{\Sigma_{1}} \tau_{\alpha 3,33} d a
$$

With the help of the equilibrium equations we can write

$$
\begin{aligned}
& \tau_{\alpha 3}=\tau_{\alpha 3}+x_{\alpha}\left(\tau_{j 3, j}-\mu_{r s 3, r s}+F_{3}\right)= \\
& =\tau_{\alpha 3}+x_{\alpha}\left(\tau_{\beta 3, \beta}-\mu_{\beta \nu 3, \beta \nu}+\tau_{33,3}-2 \mu_{3 \beta 3,3 \beta}-\mu_{333,33}+F_{3}\right)= \\
& =\left[x_{\alpha}\left(\tau_{\beta 3}-\mu_{\beta \nu 3, \nu}\right)\right]_{, \beta}+\mu_{\alpha \nu 3, \nu}+x_{\alpha}\left(\tau_{33,3}-\mu_{333,33}-2 \mu_{3 \beta 3,3 \beta}+F_{3}\right) .
\end{aligned}
$$

It is easy to show that

$$
\left(D_{k} n_{k}\right) n_{s} n_{p} n_{\beta} \mu_{s p i}-D_{j}\left(n_{r} \mu_{r j i}\right)=\left[\left(\mu_{p j i} n_{p} n_{r}-\mu_{p r i} n_{p} n_{j}\right)_{, r}\right] n_{j} .
$$

Thus, the conditions $P_{3}=\widetilde{P}_{3}$ on $\Pi$ can be written in the form

$$
\begin{gathered}
{\left[\tau_{\beta 3}-\mu_{\beta \nu 3, \nu}+\left(\mu_{\rho \beta 3} n_{\rho} n_{\eta}-\mu_{\rho \eta 3} n_{\rho} n_{\beta}\right)_{, \eta}-\right.} \\
\left.-\left(\mu_{\rho 33} n_{\rho} n_{\beta}\right)_{, 3}-\mu_{3 \beta 3,3}\right] n_{\beta}=\widetilde{P}_{3} \text { on } \Pi .
\end{gathered}
$$

It follows from (52) and (53) that

$$
\begin{aligned}
& \int_{\Sigma} \tau_{\alpha 3} d a=\int_{\Sigma} x_{\alpha} F_{3} d a+\int_{\Gamma_{1}} x_{\alpha} \widetilde{P}_{3} d s+\int_{\Gamma_{1}} x_{\alpha}\left\{\left(\mu_{\rho \nu 3} n_{\rho} n_{\beta}-\mu_{\rho \beta 3} n_{\rho} n_{\nu}\right)_{, \nu}\right] n_{\beta}+ \\
& \left.+2 \mu_{\rho 33,3} n_{\rho}\right\} d s+\int_{\Sigma}\left[\mu_{\alpha \nu, \nu}+x_{\alpha}\left(\tau_{33,3}-\mu_{333,33}-2 \mu_{3 \alpha 3,3 \alpha}\right)\right] d a
\end{aligned}
$$

If we use the relation

$$
\int_{\Sigma} x_{\alpha}\left[\left(\mu_{\rho \nu 3} n_{\rho} n_{\beta}-\mu_{\rho \beta 3} n_{\rho} n_{\nu}\right)_{, \nu}\right] n_{\beta} d s=\int_{\Gamma_{1}} n_{\alpha} \widetilde{R}_{3} d s-\int_{\Sigma} \mu_{\alpha \nu 3, \nu} d a,
$$

then (54) takes the form

$$
\int_{\Sigma} \tau_{\alpha 3} d a=\int_{\Sigma} x_{\alpha} F_{3} d a+\int_{\Gamma_{1}}\left(x_{\alpha} \widetilde{P}_{3}+n_{\alpha} \widetilde{R}_{3}\right) d s+\int_{\Sigma}\left[x_{\alpha}\left(\tau_{33,3}-\mu_{333,33}\right)+2 \mu_{\alpha 33,3}\right] d a .
$$

Since the functions $\tau_{i j}$ and $\mu_{p q r}$ are polynomials of degree two in the axial coordinate, from (13) and (55) we find that

$$
\int_{\Sigma} \tau_{\alpha 3,33} d a=0
$$


The relations (37) and (56) imply

$$
\int_{\Sigma}\left(\mu c_{4} \varepsilon_{3 \beta \alpha} x_{\beta}+2 f \varepsilon_{\alpha \rho 3} c_{\rho}+\sum_{k=1}^{4} c_{k} \tau_{\alpha 3}^{(k)}\right) d a=0 .
$$

It follows from (51) and (56) that the first two conditions from (50) are satisfied. We introduce the notations

$$
\begin{aligned}
D_{\alpha k} & =\int_{\Sigma}\left(x_{\alpha} \pi_{33}^{(k)}+2 q_{\alpha 33}^{(k)}-q_{33 \alpha}^{(k)}\right) d a, \quad D_{3 k}=\int_{\Sigma} \pi_{33}^{(k)} d a \\
D_{4 k} & =\int_{\Sigma} \varepsilon_{3 \alpha \beta}\left(x_{\alpha} \pi_{3 \beta}^{(k)}+2 q_{\alpha 3 \beta}^{(k)}\right) d a
\end{aligned}
$$

where

$$
\begin{aligned}
\pi_{33}^{(\alpha)} & =\lambda \mu_{\rho, \rho}^{(\alpha)}+(\lambda+2 \mu) x_{\alpha}, \pi_{33}^{(3)}=\lambda u_{\rho, \rho}^{(3)}+\lambda+2 \mu, \\
\pi_{33}^{(4)} & =\lambda u_{\rho, \rho}^{(4)}+4 f, \pi_{3 \beta}^{(\alpha)}=\tau_{\beta 3}^{(\alpha)}+2 f \varepsilon_{3 \beta \rho} c_{\rho}, \\
\pi_{3 \beta}^{(3)} & =\tau_{\beta 3}^{(3)}, \pi_{3 \beta}^{(4)}=\tau_{\beta 3}^{(4)}+\mu \varepsilon_{\rho \beta 3} x_{\rho}, \\
q_{\alpha 33}^{(j)} & =\frac{1}{2}\left(2 \alpha_{2}-\alpha_{1}+4 \alpha_{4}\right) \delta_{j \alpha}+\mu_{\alpha 33}^{(j)}, \quad(j=1,2,3), \\
q_{\alpha 33}^{(4)} & =-\frac{1}{2} f x_{\alpha}+\mu_{\alpha 33}^{(4)}, \\
q_{33 \alpha}^{(j)} & =\left(\alpha_{1}-2 \alpha_{3}-2 \alpha_{4}+2 \alpha_{5}\right) \delta_{j \alpha}+\mu_{33 \alpha}^{(j)}, \\
q_{33 \alpha}^{(4)} & =f x_{\alpha}+\mu_{33 \alpha}^{(4)}, q_{\alpha 3 \beta}^{(\rho)}=\varepsilon_{3 \alpha \beta} f x_{\rho}+\mu_{\alpha 3 \beta}^{(\rho)}, \\
q_{\alpha 3 \beta}^{(3)} & =\varepsilon_{3 \alpha \beta} f+\mu_{\alpha 3 \beta}^{(3)}, q_{\alpha 3 \beta}^{(4)}=\varepsilon_{3 \alpha \beta}\left(2 \alpha_{4}-\alpha_{5}\right)+\mu_{\alpha 3, \beta}^{(4)} .
\end{aligned}
$$

The constants $D_{r s},(r, s=1,2,3,4)$, can be determined after the solving of the generalized plane strain problem $A^{(k)},(k=1,2,3,4)$. From (42), (49), (51) and the divergence theorem we get

$$
\begin{aligned}
& \int_{\Sigma_{1}} G_{3}^{(1)} d a+\int_{\Gamma_{1}} \Phi_{3}^{(1)} d a=\sum_{k=1}^{4} D_{3 k} c_{k}, \\
& \int_{\Sigma_{1}} \varepsilon_{3 \alpha \beta} x_{\alpha} G_{\beta}^{(1)} d a+\int_{\Gamma_{1}} \varepsilon_{3 \alpha \beta}\left(x_{\alpha} \Phi_{\beta}^{(1)}+n_{\alpha} \Psi_{\beta}^{(1)}\right) d s=\sum_{k=1}^{4} D_{4 k} c_{k} .
\end{aligned}
$$

Thus, the remaining conditions from (50) reduce to

$$
\sum_{k=1}^{4} D_{3 k} c_{k}=0, \sum_{k=1}^{4} D_{4 k} c_{k}=0 .
$$

Let us study now the problem $\left(\mathcal{A}_{0}\right)$. The necessary and sufficient conditions to solve this problem are

$$
\int_{\Sigma_{1}} G_{j}^{(0)} d a+\int_{\Gamma_{1}} \Phi_{j}^{(0)} d s=0, \int_{\Sigma_{1}} \varepsilon_{3 \alpha \beta} x_{\alpha} G_{\beta}^{(0)} d a+\int_{\Gamma_{1}} \varepsilon_{3 \alpha \beta}\left(x_{\alpha} \Phi_{\beta}^{(1)}+n_{\alpha} \Psi_{\beta}^{(1)}\right) d s=0 .
$$


In view of (37) and (57) we obtain

$$
\int_{\Sigma} \tau_{\alpha 3,3} d a=\int_{\Sigma_{1}}\left(s_{\alpha 3}+2 f \varepsilon_{\alpha \rho 3} b_{\rho}+\mu \varepsilon_{3 \beta \alpha} b_{4} x_{\beta}+\sum_{k=1}^{4} b_{k} \tau_{\alpha 3}^{(k)}+S_{\alpha 3}\right) d a .
$$

It follows from (37), (39), (41) and (49) that

$$
\int_{\Sigma_{1}} G_{\alpha}^{(0)} d a+\int_{\Gamma_{1}} \Phi_{\alpha}^{(0)} d s=\int_{\Sigma_{1}} F_{\alpha} d a+\int_{\Gamma_{1}} \widetilde{P}_{\alpha} d s+\int_{\Sigma_{1}}\left(\tau_{\alpha 3,3}-\mu_{33 \alpha, 33}\right) d a .
$$

With the help of (55) we get

$$
\int_{\Sigma_{1}} \tau_{\alpha 3,3} d a=\int_{\Sigma_{1}}\left[x_{\alpha}\left(\tau_{33,33}-\mu_{333,333}\right)+2 \mu_{\alpha 33,33}\right] d a .
$$

By (31), (39), (58) and (65) we obtain

$$
\int_{\Sigma_{1}}\left(\tau_{\alpha 3,3}-\mu_{33 \alpha, 33}\right) d a=\sum_{k=1}^{4} D_{\alpha k} c_{k}
$$

In view of (64) and (66), the first two conditions from (62) become

$$
\sum_{k=1}^{4} D_{\alpha k} c_{k}=-\int_{\Sigma_{1}} F_{\alpha} d a-\int_{\Gamma_{1}} \widetilde{P}_{\alpha} d s
$$

The positive definiteness of the elastic potential and the reciprocal theorem imply (Ieşan, 2013)

$$
\operatorname{det}\left(D_{r s}\right) \neq 0, \quad D_{r s}=D_{s r}
$$

The system (61), (67) uniquely determines the constants $c_{s},(s=1,2,3,4)$. As the conditions (50) are satisfied we shall assume that the functions $v_{k}$ are known. From (41), (49) and (51) we get

$$
\begin{aligned}
& \int_{\Sigma_{1}} G_{3}^{(0)} d a+\int_{\Gamma_{1}} \Phi_{3}^{(0)} d s=\int_{\Sigma_{1}} F_{3} d a+\int_{\Gamma_{1}} \widetilde{P}_{3} d s+\sum_{k=1}^{4} D_{3 k} b_{k}+ \\
& +\int_{\Sigma_{1}}\left[s_{33}-\left(\alpha_{1}+2 \alpha_{3}\right) \sum_{k=1}^{4} c_{k} \kappa_{333}^{(k)}+S_{33}\right] d a \\
& \int_{\Sigma_{1}} \varepsilon_{3 \alpha \beta} x_{\alpha} G_{\beta}^{(0)} d a+\int_{\Gamma_{1}} \varepsilon_{3 \alpha \beta}\left(x_{\alpha} \Phi_{\beta}^{(0)}+n_{\alpha} \Psi_{\beta}^{(0)}\right) d s= \\
& =\int_{\Sigma_{1}} \varepsilon_{3 \alpha \beta} x_{\alpha} F_{\beta} d a+\int_{\Gamma_{1}} \varepsilon_{3 \alpha \beta}\left(x_{\alpha} \widetilde{P}_{\beta}+n_{\alpha} \widetilde{R}_{\beta}\right) d s+\sum_{k=1}^{4} D_{4 k} b_{k}+ \\
& +\int_{\Sigma_{1}} \varepsilon_{3 \alpha \beta} x_{\alpha}\left[s_{\beta 3}-\sum_{k=1}^{4} c_{k} \mu_{33 \beta}^{(k)}-\left(\alpha_{1}-2 \alpha_{3}-2 \alpha_{4}+2 \alpha_{5}\right) c_{\beta}-f c_{4} x_{\beta}+S_{\beta 3}\right] d a
\end{aligned}
$$


In view of (69) we find that the last two conditions from (62) become

$$
\begin{aligned}
& \sum_{k=1}^{4} D_{3 k} b_{k}=-\int_{\Sigma_{1}} F_{3} d s-\int_{\Gamma_{1}} \widetilde{P}_{3} d s-\int_{\Sigma_{1}}\left[s_{33}-\left(\alpha_{1}+2 \alpha_{3}\right) \sum_{k=1}^{4} c_{k} \kappa_{333}^{(k)}+S_{33}\right] d a \\
& \sum_{k=1}^{4} D_{4 k} b_{k}=-\int_{\Sigma_{1}} \varepsilon_{3 \alpha \beta} x_{\alpha} F_{\beta} d a-\int_{\Gamma_{1}} \varepsilon_{3 \alpha \beta}\left(x_{\alpha} \widetilde{P}_{\beta}+n_{\alpha} \widetilde{R}_{\beta}\right) d s- \\
& -\int_{\Sigma_{1}} \varepsilon_{3 \alpha \beta} x_{\alpha}\left[s_{\beta 3}-\left(\alpha_{1}-2 \alpha_{3}-2 \alpha_{4}+2 \alpha_{5}\right) c_{\beta}-f c_{4} x_{\beta}+S_{\beta 3}-\sum_{k=1}^{4} c_{k} \mu_{33 \beta}^{(k)}\right] d a .
\end{aligned}
$$

Let us investigate now the conditions (9). It follows from (4) that

$$
\begin{aligned}
& P_{i}=-\tau_{3 i}+2 \mu_{\alpha 3 i, \alpha}+\mu_{33 i, 3}, R_{i}=\mu_{33 i} \text { on } \Sigma_{1}, \\
& Q_{i}=-2 \mu_{\alpha 3 i} n_{\alpha} \text { on } \Gamma_{1} .
\end{aligned}
$$

Thus we have

$$
\int_{\Sigma_{1}} P_{\alpha} d a+\int_{\Gamma_{1}} Q_{\alpha}=\int_{\Sigma_{1}}\left(\tau_{3 \alpha}-\mu_{33 \alpha, 3}\right) d a
$$

If we use the relations (55) then we obtain

$$
\begin{aligned}
& \int_{\Sigma_{1}} P_{\alpha} d a+\int_{\Gamma_{1}} Q_{\alpha} d s=-\int_{\Sigma_{1}} x_{\alpha} F_{3} d a-\int_{\Gamma_{1}}\left(x_{\alpha} \widetilde{P}_{3}+n_{\alpha} \widetilde{R}_{3}\right) d s- \\
& -\int_{\Sigma_{1}}\left[x_{\alpha}\left(\tau_{33,3}-\mu_{333,33}\right)+2 \mu_{\alpha 33,3}-\mu_{33 \alpha, 3}\right] d a .
\end{aligned}
$$

With the help of (31), (39) and (51) we find that for $x_{3}=0$ we have

$$
\begin{aligned}
& \int_{\Sigma_{1}} P_{\alpha} d a+\int_{\Gamma_{1}} Q_{\alpha} d s=-\int_{\Sigma_{1}} x_{\alpha} F_{3} d a-\int_{\Gamma_{1}}\left(x_{\alpha} \widetilde{P}_{3}+n_{\alpha} \widetilde{R}_{3}\right) d s-\sum_{k=1}^{4} D_{\alpha k} b_{k}- \\
& -\int_{\Sigma_{1}}\left\{x_{\alpha}\left[s_{33}+S_{33}-\left(\alpha_{1}+2 \alpha_{3}\right) \sum_{k=1}^{4} c_{k} \kappa_{333}^{(k)}\right]+2\left(m_{\alpha 33}+M_{\alpha 33}\right)-m_{33 \alpha}-M_{33 \alpha}\right\} d a .
\end{aligned}
$$

Thus, the conditions (9) become

$$
\begin{aligned}
& \sum_{k=1}^{4} D_{\alpha k} b_{k}=-\mathcal{F}_{\alpha}-\int_{\Sigma_{1}} x_{\alpha} F_{3} d a-\int_{\Gamma_{1}}\left(x_{\alpha} \widetilde{P}_{3}+n_{\alpha} \widetilde{R}_{3}\right) d s- \\
& -\int_{\Sigma_{1}}\left\{x_{\alpha}\left[s_{33}+S_{33}-\left(\alpha_{1}+2 \alpha_{3}\right) \sum_{k=1}^{4} c_{k} \kappa_{333}^{(k)}\right]+\right. \\
& \left.+2\left(m_{\alpha 33}+M_{\alpha 33}\right)-m_{33 \alpha}-M_{33 \alpha}\right\} d a .
\end{aligned}
$$

In view of (68), the system (70), (71) uniquely determines the constants $b_{k}$, $(k=1,2,3,4)$. Since the conditions (62) are satisfied we can assume that the functions $w_{k}$ are known. Let us note that on the end located at $x_{3}=0$ we 
have

$$
\begin{aligned}
& \int_{\Sigma_{1}} P_{3} d a+\int_{\Gamma_{1}} Q_{3} d s=-\int_{\Sigma_{1}}\left(\tau_{33}-\mu_{333,3}\right) d a \\
& \int_{\Sigma_{1}}\left(x_{\alpha} P_{3}+R_{\alpha}\right) d a+\int_{\Gamma_{1}} x_{\alpha} Q_{3} d s=-\int_{\Sigma_{1}}\left[x_{\alpha}\left(\tau_{33}-\mu_{333,3}\right)+2 \mu_{\alpha 33}-\mu_{33 \alpha}\right] d a, \\
& \int_{\Sigma_{1}} \varepsilon_{\alpha \beta 3} x_{\alpha} P_{\beta} d a+\int_{\Gamma_{1}} \varepsilon_{\alpha \beta 3} x_{\alpha} Q_{\beta} d s=\int_{\Sigma_{1}}\left[\varepsilon_{\alpha \beta 3} x_{\alpha}\left(\mu_{33 \beta, 3}-\tau_{33}\right)-2 \varepsilon_{\alpha \beta 3} \mu_{a 3 \beta}\right] d a .
\end{aligned}
$$

It follows from (37), (39) and (51) that

$$
\begin{aligned}
& \int_{\Sigma_{1}} P_{3} d a+\int_{\Gamma_{1}} Q_{3} d s=-\sum_{k=1}^{4} D_{3 k} a_{k}-\mathcal{F}_{3}^{*}, \\
& \int_{\Sigma_{1}}\left(x_{\alpha} P_{3}+R_{\alpha}\right) d a+\int_{\Gamma_{1}} x_{\alpha} Q_{3} d s=-\sum_{k=1}^{4} D_{\alpha k} a_{k}-\varepsilon_{3 \alpha \beta} \mathcal{M}_{\beta}^{*}, \\
& \int_{\Sigma_{1}} \varepsilon_{\alpha \beta 3} x_{\alpha} P_{\beta} d a+\int_{\Gamma_{1}} \varepsilon_{\alpha \beta 3} x_{\alpha} Q_{\beta} d s=-\sum_{k=1}^{4} D_{4 k} a_{k}-\mathcal{M}_{3}^{*},
\end{aligned}
$$

where

$$
\begin{aligned}
\mathcal{F}_{3}^{*} & =\int_{\Sigma_{1}}\left[t_{33}+T_{33}-m_{333}-M_{333}-\left(\alpha_{1}+2 \alpha_{3}\right) \sum_{k=1}^{4} b_{k} \kappa_{333}^{(k)}\right] d a \\
\mathcal{M}_{\alpha}^{*} & =\varepsilon_{3 \alpha \beta} \int_{\Sigma_{1}}\left\{x _ { \beta } \left[t_{33}+T_{33}-m_{333}-M_{333}-\right.\right. \\
& \left.\left.-\left(\alpha_{1}+2 \alpha_{3}\right) \sum_{k=1}^{4} b_{k} \kappa_{333}^{(k)}\right]+2 \nu_{\beta 33}+2 N_{\beta 33}-\nu_{33 \beta}-N_{33 \beta}\right\} d a \\
\mathcal{M}_{3}^{*} & =\int_{\Sigma_{1}}\left\{\varepsilon _ { \alpha \beta 3 } x _ { \alpha } \left[t_{\beta 3}+T_{\beta 3}-m_{33 \beta}-M_{33 \beta}-\left(\alpha_{1}-2 \alpha_{3}-2 \alpha_{4}+2 \alpha_{5}\right) b_{\beta}-\right.\right. \\
& \left.\left.-f b_{4} x_{\beta}-\sum_{k=1}^{4} b_{k} \mu_{33 \beta}^{(k)}\right]+2 \varepsilon_{\alpha \beta 3}\left(\nu_{\alpha 3 \beta}+N_{\alpha 3 \beta}\right)\right\} d a
\end{aligned}
$$

In view of (72) the conditions (10)-(12) reduce to

$$
\begin{aligned}
& \sum_{k=1}^{4} D_{\alpha k} a_{k}=\varepsilon_{3 \alpha \beta}\left(M_{\beta}+\mathcal{M}_{\beta}^{*}\right), \\
& \sum_{k=1}^{4} D_{3 k} a_{k}=-\mathcal{F}_{3}-\mathcal{F}_{3}^{*}, \sum_{k=1}^{4} D_{4 k} a_{k}=-M_{3}-\mathcal{M}_{3}^{*} .
\end{aligned}
$$

The system (74) uniquely determines the constants $a_{j},(j=1,2,3,4)$.

Thus, the solution of the problem is given by (31). The unknown functions and unknown constants shall be determined in the following order. First, we find the solutions of the two-dimensional problems $A^{(k)},(k=1,2,3,4)$. Then, we determine the constants $D_{r s}$ from (58) and the constants $c_{k}$ from the system (61), (67). Next we determine the solution of the problem $\left(\mathcal{A}_{1}\right)$. From the system $(70),(71)$ we can calculate the constants $b_{1}, b_{2}, b_{3}$ and $b_{4}$. As the functions 
$G_{j}^{(0)}, \Phi_{j}^{(0)}$ and $\Psi_{j}^{(0)}$ are known, we can solve the problem $\left(\mathcal{A}_{0}\right)$. Finally, from (74) we determine the constants $a_{1}, a_{2}, a_{3}$ and $a_{4}$.

Remarks. 1) If $F_{\alpha}=0$ and $\widetilde{P}_{\alpha}=0$, then from (61) and (67) we find that $c_{k}=0,(k=1,2,3,4)$. By (38) and (40) we get $S_{i j}=0, M_{i j k}=0$. From (41) and (49) we obtain $G_{j}^{(1)}=0, \Phi_{j}^{(1)}=0, \Psi_{j}^{(1)}=0$ so that $v_{i}=0, s_{i j}=0$ and $m_{i j k}=0$. It follows from $(70),(71)$ and (74) that the loads $F_{3}, \widetilde{P}_{3}$ and $\widetilde{R}_{j}$ produce extension, torsion, bending, flexure and a plane deformation.

2) If $F_{j}=0, \widetilde{P}_{j}=0$ and $\widetilde{R}_{j}=0$ then we obtain the solution of Saint-Venant's problem. We note that, in contrast with the classical elasticity, the flexure of chiral bars produces extension and bending effects.

\section{Deformation of a circular cylinder subjected to a lateral pressure}

In this section we use the method presented in Section 4 to investigate the deformation of a circular cylinder subjected to a constant pressure. Let us assume that the cylinder $B$ is defined by $B=\left\{x: x_{1}^{2}+x_{2}^{2}<a^{2}, 0<x_{3}<h\right\}$, $a>0$. We suppose that the cylinder is subjected to the following loads

$$
F_{j}=0, \widetilde{P}_{\alpha}=-P n_{\alpha}, \widetilde{P}_{3}=0, \widetilde{R}_{j}=0, \mathcal{F}_{j}=0, M_{j}=0,
$$

where $P$ is a given positive constant. It is easy to see that the conditions (7) for the existence of a solution are satisfied. In this case we have

$$
\int_{\Sigma_{1}} F_{\alpha} d a+\int_{\Gamma_{1}} \widetilde{P}_{\alpha} d s=0
$$

so that the system (61), (67) implies that $c_{k}=0,(k=1,2,3,4)$. From (38), (40), (41) and (49) we find $S_{i j}=0, M_{i j k}=0, G_{j}^{(1)}=0, \Phi_{j}^{(1)}=0, \Psi_{j}^{(1)}=0$. We conclude that the solution of the problem $\left(\mathcal{A}_{1}\right)$ is $v_{j}$, so that $s_{i j}=0, m_{i j k}=0$. It follows from $(70)$ and $(71)$ that $b_{k}=0,(k=1,2,3,4)$. Thus, to find the solution (31) we have to determine the functions $w_{j}$, to solve the auxiliary two-dimensional problems $A^{(k)},(k=1,2,3,4)$, and to calculate the constants $a_{k}$. In what follows we shall use the notation $r=\left(x_{1}^{2}+x_{2}^{2}\right)^{1 / 2}$. Clearly, on the boundary of $\Sigma$ we have $r=a$ and $n_{\alpha}=x_{\alpha} / a$. Let us study the problem $\left(\mathcal{A}_{0}\right)$. From (38), (40), (41), (46), (49) and (75) we find that

$$
G_{j}^{(0)}=0, \Phi_{\alpha}^{(0)}=-P n_{\alpha}, \Phi_{3}^{(0)}=0, \Psi_{j}^{(0)}=0
$$

We seek the solution of the problem $\left(\mathcal{A}_{0}\right)$ in the form

$$
w_{\alpha}=x_{\alpha} U(r), w_{3}=0, \text { on } \Sigma_{1},
$$


where $U$ is an unknown function. We note that

$$
\begin{aligned}
w_{\alpha, \beta} & =\delta_{\alpha \beta} U+x_{\alpha} x_{\beta} r^{-1} U^{\prime}, w_{\alpha, \beta \nu}=x_{\alpha} x_{\beta} x_{\nu} r^{-2} U^{\prime \prime}- \\
& -r^{-3} x_{\alpha} x_{\beta} x_{\nu} U^{\prime}+r^{-1} U^{\prime}\left(\delta_{\alpha \beta} x_{\nu}+\delta_{\alpha \nu} x_{\beta}+\delta_{\beta \nu} x_{\alpha}\right), \\
w_{\rho, \rho \alpha} & =x_{\alpha}\left(U^{\prime \prime}+3 r^{-1} U^{\prime}\right)=\Delta w_{\alpha},
\end{aligned}
$$

where $U^{\prime}=d U / d r, U^{\prime \prime}=d^{2} U / d r^{2}$. The equations of equilibrium (20) reduce to

$$
\left(\frac{d^{2}}{d r^{2}}+3 r^{-1} \frac{d}{d r}\right)\left(\frac{d^{2}}{d r^{2}}+3 r^{-1} \frac{d}{d r}-l_{1}^{2}\right) U=0
$$

where $l_{1}^{2}=\left[2\left(\alpha_{1}+\alpha_{2}+\alpha_{3}+\alpha_{4}+\alpha_{5}\right) /(\lambda+2 \mu)\right]^{1 / 2}$. The solution which is bounded in origin is given by

$$
U=C_{1}+C_{2} I_{1}\left(r / l_{1}\right)
$$

where $C_{1}$ and $C_{2}$ are arbitrary constants, and $I_{1}$ is the modified Bessel function of the first kind and order one. Let us investigate the boundary conditions (47). From (32), (35), (45) and (78) we find that on the boundary of $\Sigma$ we have

$$
\begin{aligned}
\Lambda_{\alpha}^{(0)} & =2 x_{\alpha}\left[\left(\alpha_{1}+\alpha_{2}+\alpha_{3}+\alpha_{4}+\alpha_{5}\right) U^{\prime \prime}+\left(3 \alpha_{1}+3 \alpha_{2}+3 \alpha_{3}+\right.\right. \\
& \left.\left.+2 \alpha_{4}+2 \alpha_{5}\right) a^{-1} U^{\prime}\right], \Lambda_{3}^{(0)}=0 .
\end{aligned}
$$

The conditions $\Lambda_{j}^{(0)}=0$ on $\Gamma_{1}$ are satisfied if $C_{2}=0$. In view of (32), (35), (46), (77) and (78) we get

$$
\Pi_{\alpha}^{(0)}=2 C_{1}(\lambda+2 \mu) n_{\alpha}, \quad \Pi_{3}^{(0)}=0 \text { on } \Gamma_{1} .
$$

It follows from (76) and (81) that the conditions (47) on $\Gamma_{1}$ are satisfied if

$$
C_{1}=-\frac{P}{2(\lambda+2 \mu)}
$$

In this case, from (73) we obtain

$$
\mathcal{F}_{3}^{*}=-\pi a^{2} \lambda P /(\lambda+\mu), \mathcal{M}_{\alpha}^{*}=0, \mathcal{M}_{3}^{*}=2 \pi f a^{2} P /(\lambda+\mu) .
$$

The method used to solve the problem $\left(\mathcal{A}_{0}\right)$ can be applied to derive the solutions to the problems $A^{(3)}$ and $A^{(4)}$. Thus, we find that the solutions of these problems are

$$
u_{\alpha}^{(3)}=-\lambda x_{\alpha} /[2(\lambda+\mu)], u_{3}^{(3)}=0, u_{\alpha}^{(4)}=f x_{\alpha} /(\lambda+\mu), u_{3}^{(4)}=0 .
$$

It follows from (84), (59) and (58) that

$$
\begin{aligned}
& D_{\alpha 3}=D_{\alpha 4}=0, D_{33}=\pi a^{2} \mu(3 \lambda+2 \mu) /(\lambda+\mu), \\
& D_{34}=D_{43}=2 \pi a^{2} f(3 \lambda+2 \mu) /(\lambda+\mu), \\
& D_{44}=\frac{1}{2} \pi a^{4} \mu+4\left(2 \alpha_{4}-\alpha_{5}-\frac{f^{2}}{\lambda+\mu}\right) \pi a^{2} .
\end{aligned}
$$


From (68) and (85) we obtain $D_{3 \alpha}=0$ and $D_{4 \alpha}=0$. In view of (75) and (83), the system (74) becomes

$$
D_{\alpha \rho} a_{\rho}=0, \quad D_{33} a_{3}+D_{34} a_{4}=-\mathcal{F}_{3}^{*}, D_{43} a_{3}+D_{44} a_{4}=-\mathcal{M}_{3}^{*} .
$$

We obtain

$$
a_{1}=a_{2}=0, a_{3}=\left(D_{34} \mathcal{M}_{3}^{*}-D_{44} \mathcal{F}_{3}^{*}\right) / \delta, a_{4}=\left(D_{34} \mathcal{F}_{3}^{*}-D_{33} \mathcal{M}_{3}^{*}\right) / \delta,
$$

where $\delta=D_{33} D_{44}-D_{34}^{2}$. We conclude from (31), (77), (84) and (86) that the solution of the problem is

$$
u_{\alpha}=\varepsilon_{3 \beta \alpha} a_{4} x_{\beta} x_{3}+\frac{1}{2(\lambda+\mu)}\left(2 f a_{4}-\lambda a_{3}\right) x_{\alpha}-\frac{P x_{\alpha}}{2(\lambda+\mu)}, u_{3}=a_{3} x_{3} .
$$

The lateral pressure $P$ generates an extension, a torsion and a plane deformation. In contrast with the case of achiral cylinders, a constant pressure acting on the lateral surface of a chiral circular cylinder produces a twist around its axis.

The cartesian coordinate frame consists of the orthonormal basis $\left(\boldsymbol{e}_{1}, \boldsymbol{e}_{2}, \boldsymbol{e}_{3}\right)$ and the origin $O$. The displacement vector $\boldsymbol{u}$ from (88) has the form

$\boldsymbol{u}=\boldsymbol{u}^{*}+\boldsymbol{u}^{(p)}+\boldsymbol{u}^{(a)}, \boldsymbol{u}^{*}=a_{4}\left(x_{1} \boldsymbol{e}_{2}-x_{2} \boldsymbol{e}_{1}\right) x_{3}, \boldsymbol{u}^{(p)}=K x_{\alpha} \boldsymbol{e}_{\alpha}, \boldsymbol{u}^{(a)}=a_{3} x_{3} \boldsymbol{e}_{3}$,

where

$$
K=\frac{1}{2(\lambda+\mu)}\left(2 f a_{4}-\lambda a_{3}-P\right)
$$

The vector $\boldsymbol{u}^{*}$ represents a rotation about $x_{3}$-axis of constant magnitude $a_{4}$ radians per unit length. The vector $\boldsymbol{u}^{(p)}$ is a plane displacement parallel to $x_{1} O x_{2}$-plane, and $\boldsymbol{u}^{(a)}$ is an axial displacement.

It follows from (83) and (87) that the constants $a_{3}$ and $a_{4}$ can be expressed in the form

$$
a_{3}=P k_{3}, \quad a_{4}=-f P k_{4},
$$

where

$$
\begin{aligned}
& k_{3}=\frac{\pi a^{2}}{(\lambda+\mu) \delta}\left(2 f^{2} D_{34}^{*}+\lambda D_{44}\right), \quad D_{34}^{*}=\frac{2 \pi a^{2}(3 \lambda+2 \mu)}{\lambda+\mu}, \\
& k_{4}=\frac{\pi a^{2}}{\delta(\lambda+\mu)}\left(\lambda D_{34}^{*}+2 D_{33}\right) .
\end{aligned}
$$

In the case of a chiral material the solution contains new terms corresponding to the material parameter $f$. Let us assume that the chiral parameter $f$ changes the sign. From (85)-(92) we see that $D_{33}, D_{44}, D_{3}^{*}, \delta, k_{3}, k_{4}, K$ and $a_{3}$ are invariant to this transformation. The coefficient $a_{4}$ given by (91) changes the sign. The presence of the chiral parameter $f$ discriminates between the two directions of rotation. In the case of an achiral material we have $a_{4}=0$. 
Let $x_{i}$ be the coordinates of the point $X$ in the reference configuration $B$, and let $y_{j}$ be the coordinates of the corresponding point $Y$ in the deformed configuration. From (88) and (89) we obtain

$$
y_{\alpha}=(1+K) x_{\alpha}+a_{4} \varepsilon_{3 \beta \alpha} x_{\beta} x_{3}, \quad y_{3}=\left(1+a_{3}\right) x_{2} .
$$

We note that

$$
y_{1}^{2}+y_{2}^{2}=\left[(1+K)^{2}+a_{4}^{2} x_{3}^{2}\right]\left(x_{1}^{2}+x_{2}^{2}\right) .
$$

Let us consider the circle $(\mathcal{L})$ of radius $\rho$, located at the plane $x_{3}=k$ in the reference state, where $\rho$ and $k$ are positive constants. We see that the image of $(\mathcal{L})$ in the deformed configuration is the circle

$$
y_{1}^{2}+y_{2}^{2}=\left[(1+K)^{2}+a_{4}^{2} k^{2}\right] \rho^{2}, \quad y_{3}=\left(1+a_{3}\right) k .
$$

Thus, the image of the circle of radius $a$, located in the plane $x_{3}=c$, is a circle of radius $R(c)$, located in the plane $y_{3}=\left(1+a_{3}\right) c$, where

$$
R(c)=a\left[(1+K)^{2}+a_{4}^{2} c^{2}\right]^{1 / 2} .
$$

The variation of radius $R(c)$ with respect to the variable $c$ is given in Fig. 1 . The material parameters used are $\lambda=7000, \mu=3000, \alpha_{j}=0, \alpha_{4}=\alpha_{5}=$ 0,002 and $f=2$ (cf. Papanicolopulos, 2011). We assumed that $a=0,04$, $h=0,5$ and $P=100$.

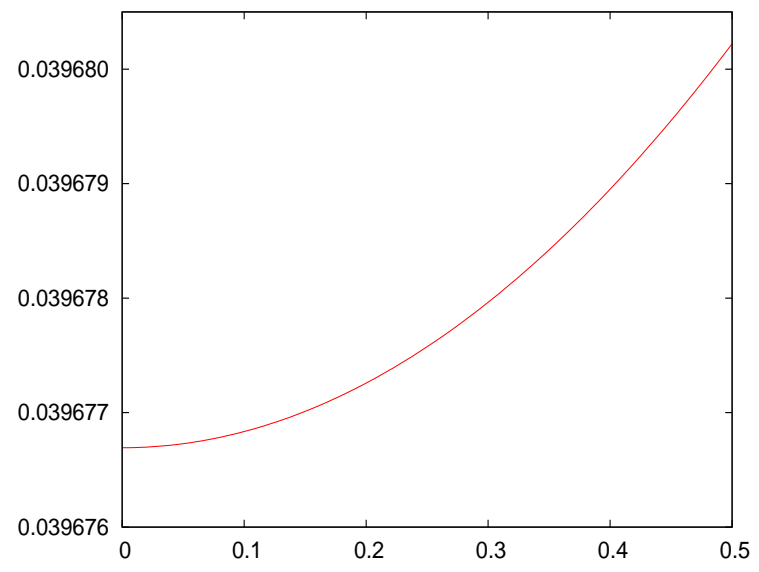

Fig. 1. Variation of $R(c)$

The solutions (84) to the problems $A^{(3)}$ and $A^{(4)}$ can be used to study other deformations of the circular cylinder. Let us consider first the problem of extension. In this case the body forces are absent $\left(F_{j}=0\right)$ and the lateral surface is free of tractions $\left(\widetilde{P}_{i}=0\right.$ and $\left.\widetilde{R}_{i}=0\right)$. The cylinder is subjected to an axial force so that $\mathcal{F}_{\alpha}=0$ and $M_{j}=0$. Let $\mathcal{F}_{3}=-F$, where $F$ is a given positive constant. In this case the resultant force of the traction acting on the end located at $x_{3}=h$ is $F \boldsymbol{e}_{3}$. From (61), (67), (70) and (71) we obtain $c_{k}=0$, $b_{k}=0,(k=1,2,3,4)$, so that the functions $w_{j}$ and $v_{j}$ which appear in (31) 
are equal to zero. In this case we have $\mathcal{F}_{j}^{*}=0, \mathcal{M}_{j}^{*}=0$ and the system (74) reduces to

$$
D_{\alpha \beta} a_{\beta}=0, D_{33} a_{3}+D_{34} a_{4}=F, \quad D_{34} a_{3}+D_{44} a_{4}=0 .
$$

Thus, we obtain

$$
a_{\alpha}=0, a_{3}=D_{44} F / \delta, a_{4}=-D_{34} F / \delta
$$

It follows from (31), (84) and (95) that the coordinates $y_{i}$ of the point $Y$ from the deformed configuration are given by

$$
y_{\alpha}=\left[1+\frac{1}{2(\lambda+\mu)}\left(2 f a_{4}-\lambda a_{3}\right)\right] x_{\alpha}+a_{4} \varepsilon_{3 \beta \alpha} x_{\beta} x_{3}, y_{3}=\left(1+a_{3}\right) x_{3} .
$$

We note that the point $O$ is fixed and that the end $\Sigma_{2}$ is subjected to the axial force $F \boldsymbol{e}_{3}$. The point $X_{0}$ which prior to deformation, had the coordinates $(0,0, h)$, goes into point $\left(0,0, h_{1}\right)$ from the deformed configuration, where

$$
h_{1}=\left(1+a_{3}\right) h
$$

In contrast with the case of an achiral material, an axial force acting on a chiral cylinder produces a torsional effect.

From (96) we obtain the following form of displacement vector in cylindrical coordinates

$$
u_{r}=\frac{1}{2(\lambda+\mu)}\left(2 f a_{4}-\lambda a_{3}\right) r, u_{\theta}=a_{4} r z, u_{z}=a_{3} z .
$$

The variation of the radial displacement with respect to the cylindrical coordinate $r$ is presented in Fig. 2. The material parameters are the same as in the previous example and we assumed that $\mathcal{F}=-\boldsymbol{e}_{3}$. The applicability of the results to specific structures requires extensive experimental verification.

Let us consider now the problem of torsion of a chiral circular cylinder. The solution to this problem can be obtained from (31) if we take into account the following conditions which characterize the torsion

$$
F_{i}=0, \widetilde{P}_{i}=0, \widetilde{R}_{i}=0, \mathcal{F}_{i}=0, M_{\alpha}=0, M_{3} \neq 0 .
$$

It is easy to see that $b_{k}=c_{k}=0,(k=1,2,3,4)$, and that the solutions of the problems $\left(\mathcal{A}_{0}\right)$ and $\left(\mathcal{A}_{1}\right)$ are equal to zero. From $(73)$ we find $\mathcal{F}_{j}^{*}=0$ and $\mathcal{M}_{j}^{*}=0$. The system $(74)$ reduces to

$$
D_{\alpha \beta} a_{\beta}=0, D_{33} a_{3}+D_{34} a_{4}=0, D_{34} a_{3}+D_{44} a_{4}=-M_{3},
$$

so that

$$
a_{1}=0, a_{2}=0, a_{3}=\frac{D_{34}}{\delta} M_{3}, a_{4}=-\frac{D_{33}}{\delta} M_{3}
$$




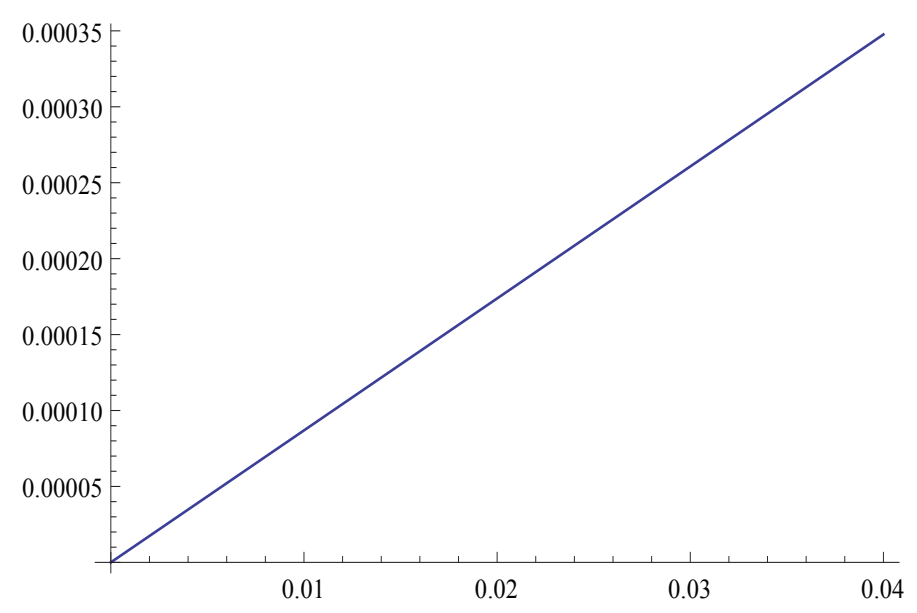

Fig. 2. Radial displacement dependence of $r$

From (31), (84) and (99) we obtain the displacement vector in the form

$$
u_{\alpha}=a_{4} \varepsilon_{3 \beta \alpha} x_{\beta} x_{3}+H x_{\alpha}, u_{3}=a_{3} x_{3},
$$

where

$$
H=\left(2 f a_{4}-\lambda a_{3}\right) / 2(\lambda+\mu) .
$$

We conclude that the torsion of a chiral cylinder generates a rotation about $x_{3}$ axis of constant magnitude $a_{4}$ radians per unit length, an axial displacement, and a radial deformation. In the case of an achiral material $(f=0)$ we have $D_{34}=0$ and $\delta=D_{33} D_{44}^{0}$ so that we obtain

$$
a_{3}=0, a_{4}=a_{4}^{0}=-\frac{1}{D_{44}^{0}} M_{3}, H=0,
$$

where

$$
D_{44}^{0}=\frac{1}{2} \pi a^{4} \mu+4\left(2 \alpha_{4}-\alpha_{5}\right) \pi a^{2} .
$$

Thus we obtain the following solution of torsion problem established by Lardner (1971) in the case of achiral materials

$$
u_{\alpha}=a_{4}^{0} \varepsilon_{3 \beta \alpha} x_{\beta} x_{3}, \quad u_{3}=0 .
$$

We note that, in contrast with the case of achiral materials, the torsion of a chiral cylinder produces an axial displacement.

In the context of the gradient elasticity of achiral materials, the deformation of circular cylinders has been studied in various papers (Lardner, 1971; Lomakin, 1987, Kahrobaiyan et al., 2011 and references therein). The gradient elasticity proposed by Altan and Aifantis (1997) has been used by Sadeghi et al. (2012) to investigate the behaviour of functionally graded micro-cylinders. 


\section{Conclusions}

The result presented in this paper can be summarized as follows:

(a) In the context of the strain gradient theory of elasticity we study the deformation of a chiral cylinder subjected to body forces, to tractions on the lateral surface and to resultant forces and moments on the ends. The three-dimensional problem is reduced to the study of some two-dimensional problems.

(b) The body forces and the tractions on the lateral surface produce extension, flexure, torsion, bending by terminal couples and a plane strain.

(c) As a special case of the problem we obtain the solution of the problem of flexure by a transversal force. In contrast with the case of achiral solids, the flexure of a chiral cylinder of arbitrary cross-section is accompanied by extension and bending by terminal couples.

(d) We use the method to study the deformation of a right circular cylinder subjected to a constant pressure on the lateral surface.

(e) We show that a uniform pressure on the lateral surface of a chiral circular cylinder does not produce bending effects.

(f) The salient feature of the solution is that a constant pressure acting on the lateral surface of a chiral circular cylinder produces a twist around its axis.

\section{Acknowledgments}

The authors thank the referees for their helpful suggestions. R.Q. is supported by the Project "Análisis Matemático de las Ecuaciones en Derivada Parciales de la Termomecánica“ (MTM2013-42004-P) of the Spanish Ministry of Economy and Competitiveness.

\section{References}

Altan, B.S., Aifantis, E.C., 1997. On some aspects in the special theory of gradient elasticity. Journal of Mechanical Behaviour of Materials, 8, 231282.

Askes, H., Aifantis, E.C., 2009. Gradient elasticity and flexural wave dispersion in carbon nanotubes. Phys. Rev. B 80, 1-8. 
Chandraseker, K., Mukherjee, S., Paci, J.T., Schatz, G.C. (2009). An atomistic continuum Cosserat rod model of carbon nanotubes. J. Mech. Phys. Solids 57, 932-958.

Hlavacek, I., Hlavacek, M., 1969. On the existence and uniqueness of solution and some variational principles in linear theories of elasticity with couple stresses. Apl. Mat., 14, 411-427.

Ieşan, D., 1986a. On SaintVenants problem. Arch. Ration. Mech. Anal. 91, 363-373.

Ieşan, D., 1986b. On the theory of uniformly loaded cyliners. J. Elasticity, 16, 375-382.

Ieşan, D., 2009. Classical and Generalized Models of Elastic Rods. Chapman \& Hall/CRC Press, New York.

Ieşan, D., 2013. On the torsion of chiral bars in gradient elasticity. Int. J. Solids Struct.

Ieşan, D., Quintanilla, R., 2007. On the deformation of inhomogeneous orthotropic elastic cylinders, Eur. J. Mech. A/Solids, 26, 999-1015.

Kahrobaiyan, M.H., Tajalli, S.A., Movahhedy, M.R., Akbari, J.,Ahmadian, M.T.,2011. Torsion of strain gradient bars. Int. J. Eng. Sci. 49, 856-866.

Khatiashvili, G.M., 1983. Almansi-Michell problem for homogeneous and composed bodies, Izd. Metzniereba, Tbilisi (in Russian).

Lakes, R.S, Yoon, H.S., Katz, J.L., 1983. Slow compressional wave propagation in wet human and bovine cortical bone. Science 200, 513-515.

Lakes, R.S, 2001. Elastic and viscoelastic behaviour of chiral materials. Int. J. Mech. Sci. 43, 1579-1589.

Lardner, R.W., 1971. Dislocations in materials with couple stress. J. Inst. Math. Appl.,7, 126-137.

Lomakin, V.A., 1987. Problems of generalized theory of elasticity with couplestresses (in Russian).Vestnik Mosk.Univ. 1, 82-88.

Mindlin, R.D., 1964. Micro-structure in linear elasticity. Arch. Ration. Mech. Anal.16, 51-78.

Mindlin, R.D., Eshel, N.N., 1968. On first strain-gradient theories in linear elasticity. Int. J. Solids Struct. 4, 109-124.

Papanicolopulos, S.A., 2011.Chirality in isotropic linear gradient elasticity. Int. J. Solids Struct. 48, 745-752.

Park, H.C., Lakes, R.S., 1986. Cosserat micromechanics of human bone: strain redistribution by a hydration-sensitive constituent. J. Biomechanics 19, 1038-1040.

Sadeghi, H., Baghani, M., Naghdabadi, R., 2012. Strain gradient elasticity solution for functionally graded micro-cylinders. Int. J. Eng. Sci. 50, 22-30.

Spadoni, A., Ruzzene, M., 2012. Elasto-static micropolar behaviour of a chiral auxetic lattice. J. Mech. Phys. Solids 60, 156-171.

Toupin, R.A., 1962. Elastic materials with couple stresses. Arch. Ration. Mech. Anal. 11, 385-414.

Wang, Q., Wang, C.M., 2007. The constitutive relation and small scale parameter of nonlocal continuum mechanics for modelling carbon nanotubes. 
Nanotechnology 18, 075702.

Zhang, Y., Wang, C., Xiang, Y., 2010. A molecular dynamics investigation of the torsional responses of defective single-walled carbon nanotubes. Carbon 48, 4100-4108. 